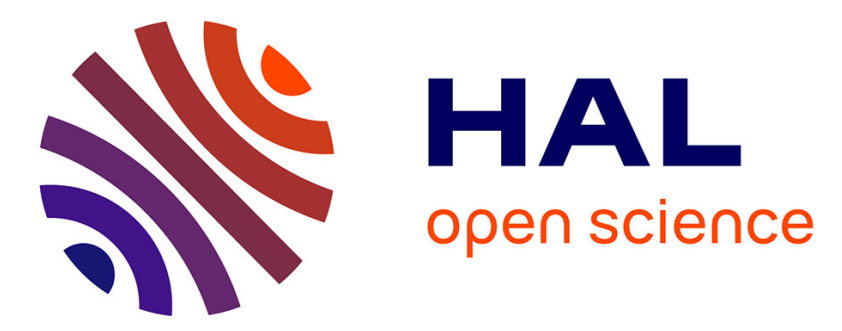

\title{
Alimentation, pathologie, reproduction et productivité de la vache laitière: Interrelations à l'échelle de la lactation et de la carrière
}

\author{
J.B. Coulon, E Landais, J.P. Garel
}

\section{- To cite this version:}

J.B. Coulon, E Landais, J.P. Garel. Alimentation, pathologie, reproduction et productivité de la vache laitière: Interrelations à l'échelle de la lactation et de la carrière. Productions Animales, 1989, 2 (3), pp.171-188. hal-00895865

\section{HAL Id: hal-00895865 https://hal.science/hal-00895865}

Submitted on 1 Jan 1989

HAL is a multi-disciplinary open access archive for the deposit and dissemination of scientific research documents, whether they are published or not. The documents may come from teaching and research institutions in France or abroad, or from public or private research centers.
L'archive ouverte pluridisciplinaire HAL, est destinée au dépôt et à la diffusion de documents scientifiques de niveau recherche, publiés ou non, émanant des établissements d'enseignement et de recherche français ou étrangers, des laboratoires publics ou privés. 
INRA Prod. Anim., 1989, 2 (3), 171-188

\section{J.B. COULON, E. LANDAIS*, J.P. GAREL**}

INRA Laboratoire de la Lactation Theix 63122 Saint-Genès-Champanelle

* INRA Unité de Recherches sur les Systèmes Agraires

et Développement, Route de St-Cyr, 78000 Versailles

** INRA Domaine de La Borie 15190 Marcenat

\section{Alimentation, pathologie, reproduction et productivité de la vache laitière}

\author{
Interrelations à l'échelle \\ de la lactation et de la carrière
}

\section{Les mammites et les boiteries sont les problèmes sanitaires les plus fréquents chez la vache laitière. Les conséquences de ces pathologies sont parfois très importantes, non seulement sur la production laitière mais aussi, d'une manière plus générale, sur la longévité de l'animal.}

\section{Résumé}

L'objectif de cette étude était de préciser l'effet des principales affections sanitaires sur la production laitière et leurs liaisons avec les caractéristiques des lactations et des carrières des animaux. L'étude a été réalisée au cours d'un essai d'alimentation (comparaison de rations à base de foin ou d'ensilage d'herbe complémentées à 2 niveaux énergétiques) de longue durée ( 6 ans) et a porté sur 487 lactations de 190 vaches Pie-Noires et Montbéliardes.

Au cours de ces 487 lactations, 595 troubles sanitaires ont été observés. Ces troubles ont donc touché $59 \%$ des lactations étudiées avec une incidence moyenne de 2,1 affections par lactation atteinte. Les boiteries et les mammites ont représenté respectivement 52 et $24 \%$ des affections. $47 \%$ des mammites ont lieu au cours des 2 premiers mois de lactation. La fréquence des boiteries augmente au cours de l'hiver et est maximale à la mise à l'herbe. A court terme (5 semaines) les pertes de production les plus importantes sont causées par les mammites hivernales $(24 \mathrm{~kg}$ ) et surtout les boiteries survenant à la mise à l'herbe $(56 \mathrm{~kg})$. A l'échelle de la lactation complète, ce sont les boiteries répétées qui entraînent les pertes de production les plus élevées : $640 \mathrm{~kg}$ de lait pour les vaches ayant présenté 3 boiteries et plus contre $20 \mathrm{~kg}$ pour celles n'ayant présenté qu'une seule boiterie et $160 \mathrm{~kg}$ pour celles ayant présenté une ou plusieurs mammites. Les boiteries répétées sont 3 fois plus fréquentes chez les vaches Pie-Noires que chez les vaches Montbéliardes et quatre fois plus fréquentes chez celles ayant reçu une ration hivernale à base d'ensilage d'herbe que chez celles ayant reçu une ration hivernale à base de foin. Quatre principales classes de lactation ont pu être définies à partir du niveau de production des animaux, de leur santé, de leur reproduction et de leur réforme. Les écarts de production entre ces 4 groupes atteignent $1800 \mathrm{~kg}$ de lait par lactation. Les vaches recevant une ration à base d'ensilage d'herbe faiblement complémentée ont eu une carrière plus courte que celles recevant les autres rations $(2,5$ lactations contre 3,2 à 3,5$)$. Les vaches présentant en première lactation des troubles sanitaires graves (boiteries multiples) ont eu une carrière plus courte de 1,1 lactation que les vaches indemnes en première lactation. En moyenne, la production laitière et le poids vif ont augmenté de respectivement 622 et $51 \mathrm{~kg}$ entre la $1^{\text {re }}$ et la $3^{\mathrm{e}}$ lactation. Cette augmentation de production laitière a été plus importante chez les vaches recevant une alimentation hivernale à base de foin (complémenté à haut ou bas niveau) ou d'ensilage d'herbe complémenté à haut niveau $(+752 \mathrm{~kg}$ ) que chez celles recevant une ration à base d'ensilage d'herbe complémenté à bas niveau $(+359 \mathrm{~kg})$. A l'échelle de 3 lactations, l'écart de production laitière entre les lots extrêmes (foin haut et ensilage bas) atteint $2770 \mathrm{~kg}$, soit $26 \%$. Ces résultats conduisent à reconsidérer certaines conclusions issues de résultats obtenus à l'échelle de l'hiver ou de la lactation.
De très nombreux travaux ont été entrepris pour analyser à court terme l'effet sur la production laitière des facteurs alimentaires (type de ration de base, modalités de distribution; nature, composition, qualité et répartition du concentré, etc). Ils ont permis de mettre en évidence et d'analyser les mécanismes mis en jeu dans l'ingestion et la digestion des aliments, de hiérarchiser les facteurs de variation en cause, d'évaluer les effets de l'alimentation sur les performances, et de proposer des principes et des méthodes opérationnelles de rationnement des animaux.

Beaucoup moins d'études ont été réalisées à l'échelle de plusieurs lactations successives et rares sont celles qui prennent en compte l'état sanitaire, la reproduction et la réforme des vaches laitières. A long terme, ces facteurs sont pourtant déterminants pour les performances de production (Broster et Broster 1984). Cette approche nécessite en effet la mise en oeuvre d'expérimentations de longue durée, impliquant un nombre élevé d'animaux et des moyens importants.

Un essai de ce type a été réalisé, de 1974 à 1980, au domaine INRA de Marcenat (Cantal(1)) et a fait l'objet d'une interprétation privilégiant la relation qui existe, à l'échelle de la lactation, entre les performances de production et les traitements expérimentaux (Coulon et al 1985). Ces données ont ensuite été analysées sous un angle plus large avec l'objectif :

- d'une part, de décrire la pathologie observée durant l'essai et d'évaluer ses effets à court, moyen et long terme sur la production laitière, ainsi que ses conséquences pour la carrière des animaux ;

(1) $1100 \mathrm{~m}$ d'altitude - température movenne annuelle de $6,8^{\circ} \mathrm{C}$-pluviométrie annuelle de $1105 \mathrm{~mm}$, bien répartie sur l'année. 
- d'autre part, d'analyser l'influence à long terme des traitements expérimentaux sur la production, la reproduction, la santé et la longévité des vaches laitières.

Cet article résume les principales conclusions de cette étude, qui ont été exposées en détail par ailleurs (Landais et al 1989, Coulon et al $1989 \mathrm{a}$ et b). On pourra se reporter à ces publications, en particulier pour une discussion plus approfondie des résultats présentés.

\section{1 / Conduite de l'étude}

\section{1 / Conditions expérimentales}

Le principe de l'essai a consisté à suivre durant toute leur carrière des vaches laitières soumises à des traitements alimentaires hivernaux constants.

L'étude porte sur 487 lactations de 190 vaches, pour moitié de race Montbéliarde et pour moitié de race Française Frisonne Pie Noire. Ces lactations se sont réparties sur six années consécutives, au cours desquelles des primipares ont été régulièrement introduites pour renouveler le troupeau expérimental (l'âge à la première mise bas étant de 32 mois en moyenne). Les vaches, qui produisaient en moyenne $4200 \mathrm{~kg}$ de lait par lactation, vêlaient pour la plupart en début d'hiver $(42 \%$ des vêlages avant le $1^{\text {er }}$ janvier) et étaient conduites du $1^{\text {er }}$ novembre au $1^{\text {er }}$ mai en stabulation entravée, faiblement paillée ( $2 \mathrm{~kg} /$ vache/jour). $\mathrm{La}$ traite avait lieu en place, au pot trayeur. La détection des chaleurs se faisait en place jusqu'au 15 janvier. Au-delà de cette date, les vaches étaient sorties environ deux heures par jour, si les conditions météorologiques le per-

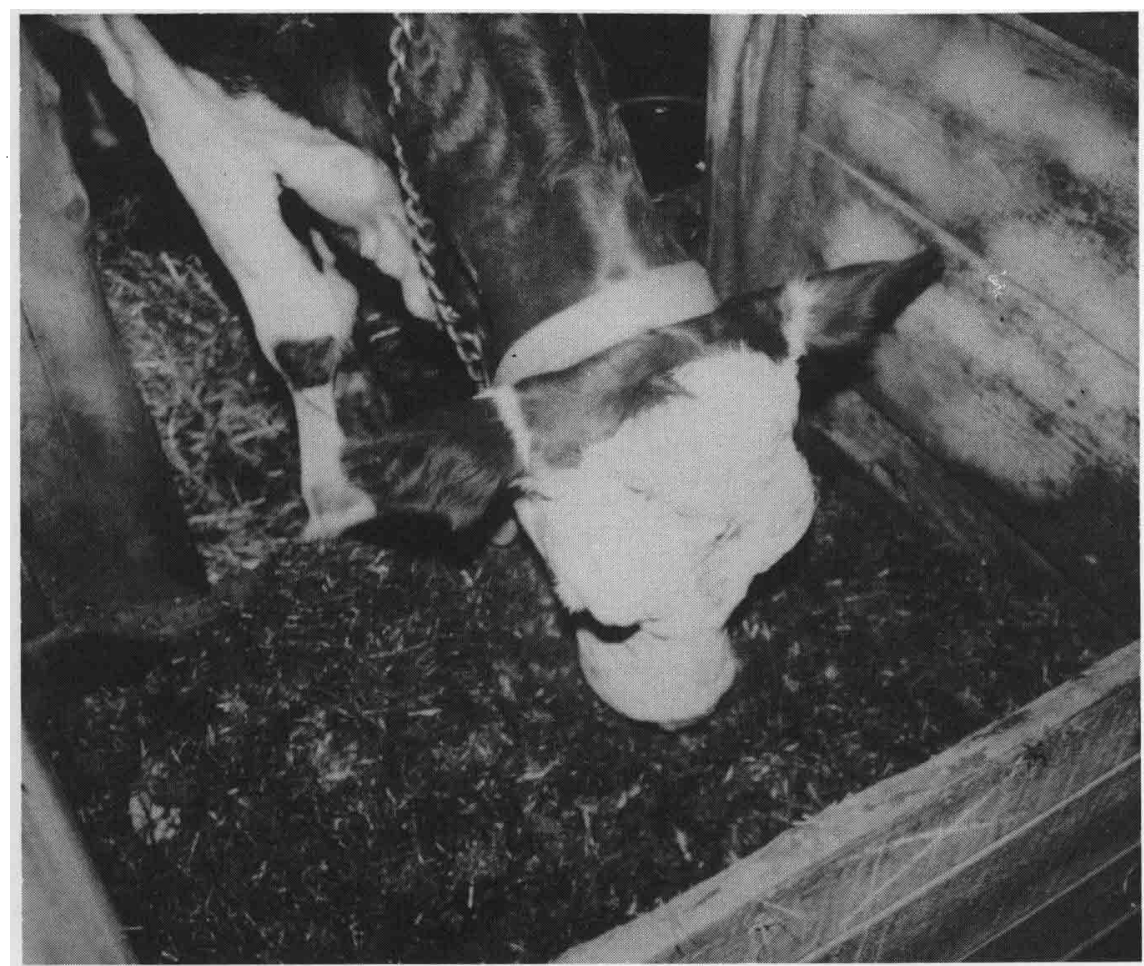

Durant les 6 années d'étude, plusieurs régimes alimentaires hivernaux ont été comparés : rations à base de foin et/ou d'ensilage complémentées à 2 niveaux énergétiques. mettaient, à partir du $45^{\mathrm{e}}$ jour suivant leur mise-bas, pour observation de leur comportement. En cas de non-retour en oestrus, des traitements ont pu leur être administrés. Les vaches recevaient, à volonté, un régime à base de foin (lots F) ou d'ensilage d'herbe de prairie naturelle (lots E). Chacune de ces rations était complémentée par un aliment concentré de type production (1 UFL et $120 \mathrm{~g}$ PDI par $\mathrm{kg}$ brut). Cette complémentation était distribuée sur la base d'un $\mathrm{kg}$ de concentré pour $2,5 \mathrm{~kg}$ de lait produit au-dessus de 5 (lots «Haut» $=\mathrm{FH}$ et $\mathrm{EH}$ ) ou de $10 \mathrm{~kg}$ de lait (lots "Bas" = FB et $\mathrm{EB})$. Un cinquième lot a été étudié, dont la ration de base était mixte $(40 \%$ de foin et $60 \%$ d'ensilage d'herbe). Cette ration n'a été testée qu'au bas niveau de complémentation (lot MB). Au pâturage, les animaux étaient conduits ensemble, en un seul troupeau, sur des parcelles situées dans un rayon de $500 \mathrm{~m}$ autour de l'étable, en système tournant. Ils étaient rentrés deux fois par jour pour la traite. Le chargement a varié de 3,5 vaches par hectare au printemps à 1,5 vache en fin d'été.

\section{2 / Relevés}

Parallèlement aux mesures zootechniques classiques (Coulon et al 1985), les troubles sanitaires ont été systématiquement relevés et regroupés en 4 grands syndromes : mammites, boiteries, troubles uro-génitaux et troubles digestifs (voir Landais et al 1989 pour le détail des relevés).

$\mathrm{Au}$ total 595 affections ont été détectées au cours des six années d'expérience. Elles ont concerné 287 des 487 lactations étudiées (soit une fréquence des "lactations atteintes" de $59 \%$ ), avec une incidence moyenne de 2,1 affections par lactation atteinte. Les principaux troubles ont concerné les membres (52\% des affections cliniques et $60 \%$ des lactations atteintes) et la mamelle (24\% des affections cliniques et $32 \%$ des lactations atteintes). Les troubles de l'appareil uro-génital ont représenté $9 \%$ des affections (rétention placentaire essentiellement), et ceux de l'appareil digestif $8 \%$.

Dans la suite du texte, l'expression « Autres troubles » désignera l'ensemble des affections autres que les mammites et les boiteries, que leur rareté ne permet pas d'étudier séparément. Dans tous les cas, deux observations d'un même trouble n'ont donné lieu à une nouvelle notation que si l'intervalle entre ces deux observations était supérieur à huit jours, et si les symptômes observés avaient disparu dans cet intervalle.

La pathologie a considérablement varié selon les années (c'est-à-dire selon les campagnes couvrant du $1^{\text {er }}$ octobre au 30 septembre) en relation notamment avec la variabilité climatique. En nous appuyant sur les taux de morbidité globale, nous avons donc regroupé, pour l'analyse de l'effet année, les campagnes comparables: années 1,2 et 4 d'une part, années 5 et 6 d'autre part. L'année 3 a été éliminée de cette analyse en raison des conditions très particulières dans lesquelles s'est déroulée la mise à l'herbe (printemps exceptionnellement humide), ce qui a entraîné une évolution atypique de la pathologie. 
Tableau 1. Incidence de la pathologie selon le rang de lactation: fréquence des lactations atteintes (en \%) et nombre moyen d'affections par lactation atteinte (entre parenthèses).

\begin{tabular}{|lccc|}
\hline Rang de lactation & 1 & 2 & 3 et plus \\
Nombre total de lactations observées & 137 & 111 & 239 \\
\hline Mammites & $20(1,78)$ & $15(1,18)$ & $21(1,51)$ \\
Boiteries & $32(1,68)$ & $24(1,89)$ & $43(1,78)$ \\
Autres troubles & $33(1,07)$ & $34(1,03)$ & $21(1,18)$ \\
\hline Total & $58(2,15)$ & $50(1,96)$ & $64(2,07)$ \\
\hline
\end{tabular}

\section{3 / Analyse des données}

Le traitement des données a mis en oeuvre des méthodes très variées, que nous avons dû adapter à la structure de l'information disponible et à la nature, relativement originale, de notre problématique. Ces méthodes sont exposées en détail dans les articles précités (Landais et al 1989, Coulon et al 1989a et b). D'une façon générale, l'un des objectifs des analyses réalisées visait à condenser l'information relative à chaque aspect de la carrière économique des vaches laitières (pathologie, production laitière, reproduction, réforme) pour les mettre ensuite en relation. Ceci a conduit à enchaîner des méthodes analytiques classiques (comparaison de fréquences et de distributions, analyses de variance) avec des méthodes plus globales (analyse multivariée et méthodes de classification typologique).

Pour l'évaluation des pertes de lait entraînées par la pathologie, nous avons tenu compte à la fois des pertes de production (mesurées directement) et des pertes de commercialisation, entraînées par l'interdiction légale de livraison consécutive aux traitements médicamenteux appliqués (évaluées à partir de la durée de suspension de la livraison, qui a été de cinq jours en moyenne).

\section{2 / Etude à l'échelle de la lactation}

\section{1 / Analyse préalable de la pathologie observée}

\section{a / Effet des facteurs fixes à l'échelle de la lactation}

\section{La race}

Les vaches Frisonnes ont été globalement près de deux fois plus touchées par la pathologie que les vaches Montbéliardes. Ceci résulte à la fois d'une plus forte proportion de lactations atteintes ( 71 contre $48 \%$ ) et d'un plus grand nombre d'affections par lactation atteinte $[2,29$ contre 1,76). Ces différences sont hautement significatives $(\mathrm{P}<0,01)$. La différence entre races est deux fois plus importante pour les boiteries que pour les mammites.

\section{Le numéro de lactation}

L'incidence de la pathologie est significativement liée au numéro de lactation, et varie selon l'affection considérée (tableau 1). Pour les mammites, comme pour les boiteries, la seconde lactation apparaît moins affectée que la première et que celles qui suivent. Les autres troubles, en revanche, atteignent un tiers des lactations de rang 1 ou 2, mais seulement un cinquième des lactations suivantes.

\section{L'alimentation}

Les régimes hivernaux n'ont eu qu'une influence modérée sur la fréquence d'apparition des troubles pathologiques. Cependant, les animaux des lots recevant de l'ensilage ont présenté un nombre moyen d'épisodes pathologiques par lactation significativement supérieur $(\mathrm{P}<0,05)$ à celui présenté par les animaux qui ne recevaient que du foin : 1,32 épisode constaté par lactation contre 1,08. Le facteur " régime " interagit d'autre part avec le facteur « rang de lactation ": alors que la fréquence des lactations atteintes évolue peu avec le rang de lactation lorsque les animaux reçoivent du foin comme ration de base, elle augmente sensiblement entre les deux premières lactations et les suivantes quand ils reçoivent de l'ensilage. Ainsi, la fréquence des lactations atteintes parmi les lactations de rang supérieur à deux est de $64 \%$ dans les lots « Ensilage» contre $49 \%$ dans les lots « Foin » $(\mathrm{P}<0,05)$.

Les animaux des lots " Haut" ont présenté, par rapport à ceux des lots «Bas", à la fois une fréquence supérieure des lactations atteintes (61 vs $56 \%$ ) et un plus grand nombre d'affections par lactation atteinte $(1,30$ vs 1,10$)$. Cet effet est imputable exclusivement aux vaches en troisième lactation et plus (chez ces animaux la fréquence des lactations atteintes est de $72 \%$ dans les lots " Haut», contre $55 \%$ dans les lots « Bas ", P $<0,05$ ).

\section{L’année}

La fréquence de l'ensemble des affections constatées a fortement diminué au cours des six années d'expérience. Cette évolution, significative pour les boiteries comme pour les mammites, a touché à la fois la fréquence des lactations atteintes (respectivement 33 et $49 \%$ pour les mammites et les boiteries en année 1 contre 14 et $18 \%$ en année 5) et le nombre moyen d'épisodes pathologiques par lactation atteinte (respectivement 1,7 et 2,2 en année 1 contre 1,3 et 1,2 en année 5 ).

\section{b / Circonstances d'apparition des troubles pathologiques}

Le stade de lactation est un facteur de variation important de la pathologie uro-génitale, ainsi qu'on pouvait s'y attendre $195 \%$ de ces troubles ont lieu au cours du premier mois de lactation). Il affecte sensiblement, d'autre part, la fréquence des mammites et des boiteries.
En 6 ans, les vaches

Frisonnes ont présenté près de 2 fois plus de troubles sanitaires que les Montbéliardes. 
Figure 1. Effet du stade de lactation sur la fréquence des mammites et des boiteries.

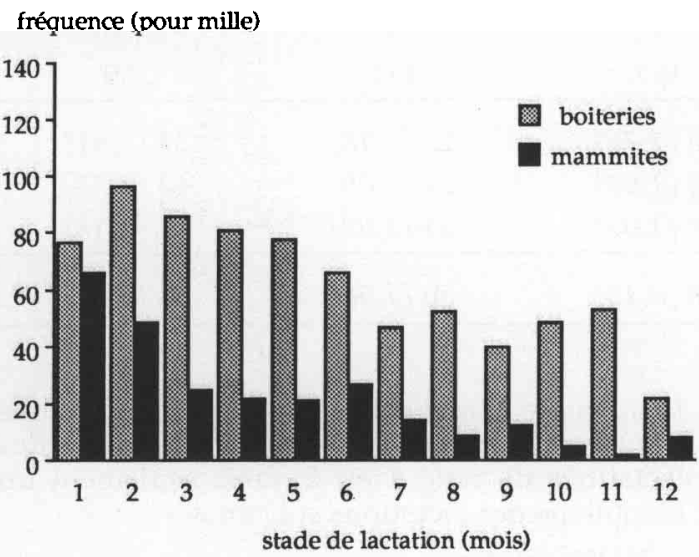

Figure 2. Effet de la saison sur la fréquence des mammites et des boiteries.
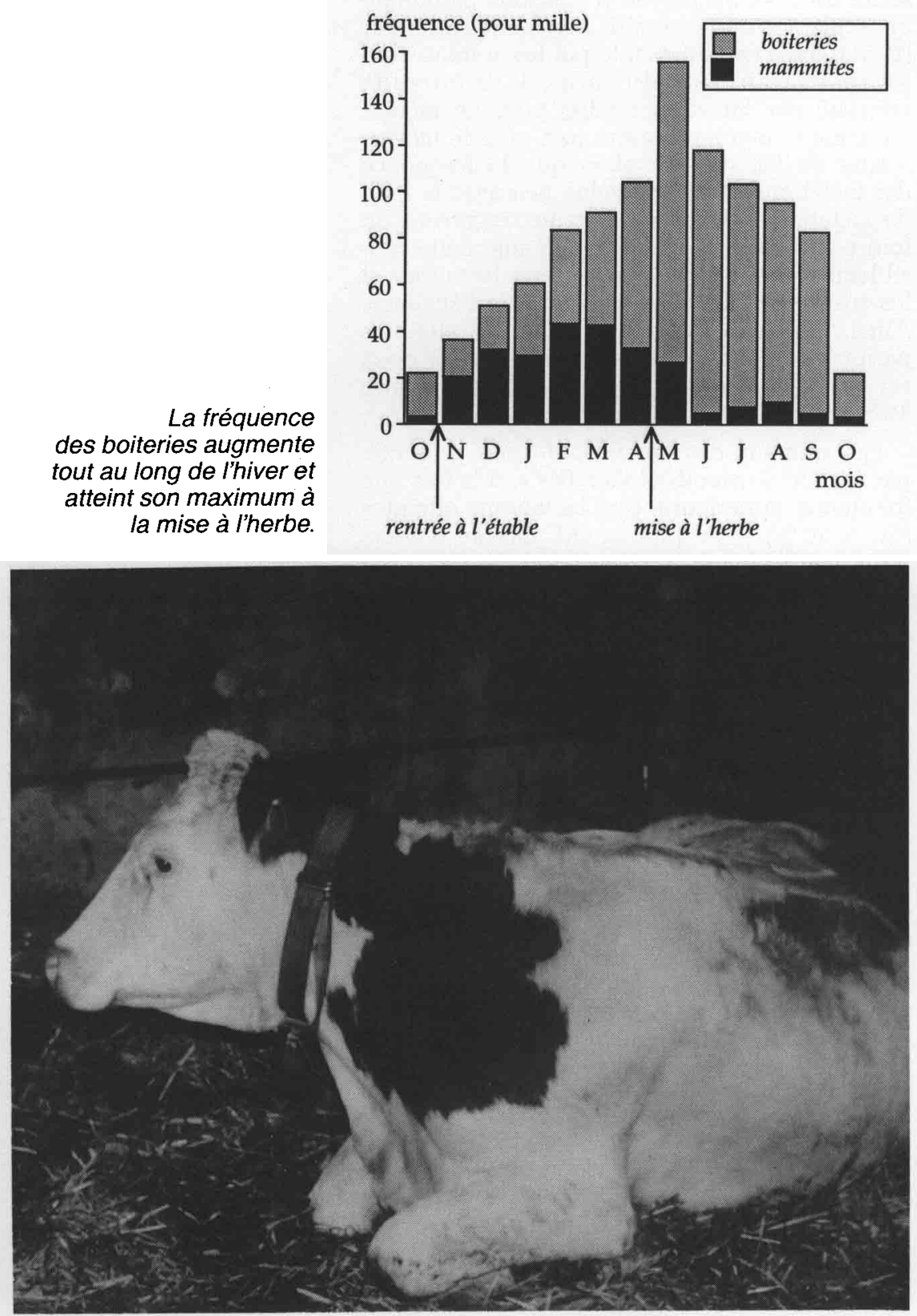

Afin de démêler ce qui revient aux effets propres du stade de lactation et de la saison, qui interagissent (en raison du groupement des vêlages en hiver), nous avons réalisé une analyse croisée, pour corriger les biais réciproques, ainsi que ceux qui sont dûs aux réformes (pour le détail de la méthode utilisée, voir Landais et al 1989). Cette analyse n'a pu être menée que sur les deux types d'affections les plus représentés, mammites et boiteries.

\section{Effets propres}

du stade de lactation (figure 1)

La fréquence des mammites, maximale dès le premier mois de lactation, décroît très rapidement au cours du second mois, puis plus lentement par la suite. L'évolution est différente pour les boiteries, dont la fréquence passe par un maximum au second mois de lactation, puis décroît irrégulièrement jusqu'à la mise bas suivante.

Effets propres de la saison (figure 2)

La rentrée à l'étable est suivie d'une augmentation sensible de la fréquence des mammites cliniques; la mise à l'herbe, à l'inverse, d'une diminution très importante de cette fréquence. Au cours de la période hivernale, la fréquence des mammites augmente irrégulièrement, passe par un maximum situé en février-mars, puis décroît dès avant la mise à l'herbe. Au cours de la période estivale, une légère recrudescence est observée après le minimum de juin, la fréquence des mammites diminuant à nouveau après le mois d'août. Au total, la fréquence des mammites est près de cinq fois plus élevée entre novembre et avril qu'entre juin et octobre.

En ce qui concerne la fréquence des boiteries, la saison hivernale est caractérisée par une augmentation continue, et la saison estivale, à l'inverse, par une diminution constante. La mise à l'herbe s'accompagne d'une augmentation brutale du nombre de boiteries enregistrées tandis que la rentrée à l'étable n'entraîne pas d'effet marqué. Au total, la conjonction de ces évolưtions contrastées entraîne un effet de relais très net entre les mammites et les boiteries.

\section{c / Association de plusieurs épisodes pathologiques au cours d'une même lactation}

\section{Répétition d'un même type d'affection}

Les récidives ont concerné $26,1 \%$ des lactations atteintes de mammite(s) et $41,6 \%$ des lactations atteintes de boiterie(s). Pour un type donné d'affection, la fréquence des récidives varie en outre selon la race et le rang de lactation (figure 3). Les boiteries, qui sont de loin l'affection la plus récidivante, le sont surtout chez les vaches Frisonnes, et c'est ce qui caractérise le mieux leur grande sensibilité à ce type d'affection. Les mammites ont également une tendance à récidiver plus marquée chez les Frisonnes que chez les Montbéliardes, mais la différence est moins sensible.

Pour les deux types d'affection considérés, la fréquence des récidives est maximale parmi les 
lactations de rang égal ou supérieur à trois, intermédiaire en première lactation, et minimale en seconde lactation.

Ces épisodes successifs sont-ils indépendants entre eux? Les différents tests qui ont été réalisés pour répondre à cette question ont clairement démontré qu'il n'en était rien. Bien au contraire, une lactation ayant présenté une mammite ou une boiterie est significativement plus exposée qu'une autre à être à nouveau affectée d'un ou de plusieurs épisodes du même trouble. Ce résultat constitue un argument fort en faveur d'une prise en compte globale de la pathologie à l'échelle de la lactation, et valide a posteriori le concept de « lactation atteinte » que nous avons utilisé.

\section{Association entre différents}

\section{types d'affections}

Dans les conditions de l'expérimentation, aucune liaison n'a pu être mise en évidence entre l'apparition des mammites, des boiteries et des «autres troubles». Ces trois types d'affections peuvent être considérés comme mutuellement indépendants.

\section{d / Typologie des " profils pathologiques de lactation »}

Les résultats qui précèdent ont permis de construire très simplement une typologie des profils pathologiques de lactation en croisant les deux principaux types de troubles rencontrés: mammites et boiteries. Nous obtenons ainsi les huit types de profils pathologiques de lactation qui sont présentés au tableau 2. Le choix d'un tableau croisé est justifié par l'indépendance entre les différents types de troubles étudiés.

Nous avons distingué trois modalités du facteur «mammite», en fonction du nombre d'épisodes constatés : 0, 1, 2 et plus. Pour les boiteries, nous avons retenu quatre modalités : $0,1,2,3$ et plus. Les " autres troubles » sont répartis uniformément dans les catégories ainsi définies, et il n'en a été tenu compte que dans la catégorie « $\mathbf{0}$ mammite, 0 boiterie », qui est subdivisée en deux sous-catégories: " autres troubles» absents (type 000) et "autres troubles » présents (type AU).
Figure 3. Répétition des pathologies au cours d'une même lactation ; effets du rang de lactation et de la race. Nombre de lactations atteintes de 1 , 2 ou 3 et + mammites (boiteries) rapporté au nombre total de lactations atteintes de mammites (boiteries).

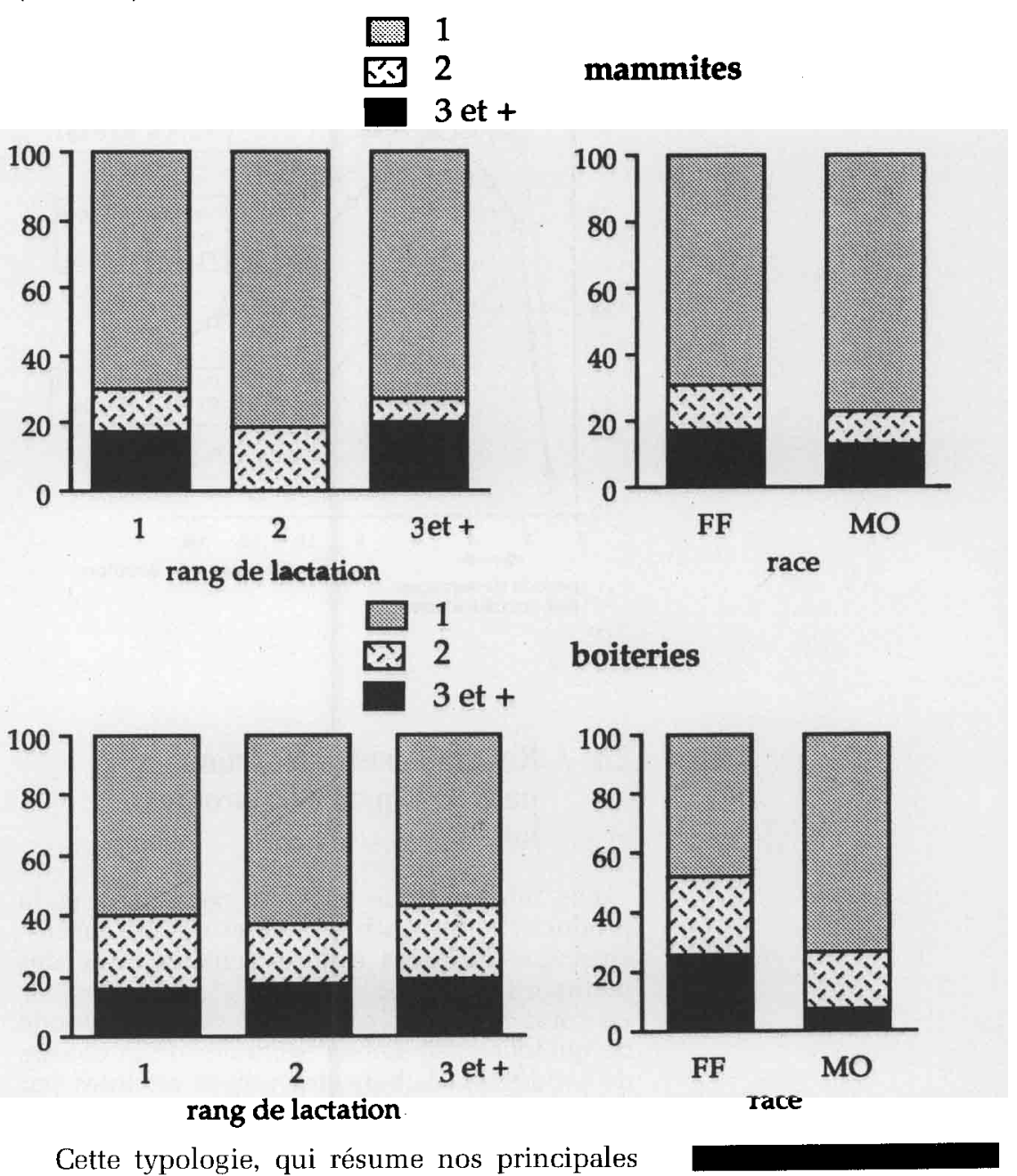

conclusions, sera utilisée par la suite pour étudier les relations entre la pathologie et la production laitière. Pour les comparaisons entre différents profils pathologiques de lactation, le témoin sera constitué par une moyenne pondérée des types 000 et $\mathrm{Au}$, de manière à évaluer sans biais les caractéristiques de chaque profil.

\section{Les récidives sont très fréquentes : plus de $40 \%$ des vaches atteintes d'une boiterie ont été affectées d'une ou plusieurs autres boiteries au cours de la même lactation.}

Tableau 2. Typologie des profils pathologiques de lactation (huit types pour $n=478$ lactations).

\begin{tabular}{|l|c|c|c|}
\hline & $\mathbf{0}$ & $\mathbf{1}$ & Mammites et plus \\
\hline $\begin{array}{l}\text { Boiteries } \\
\mathbf{0}\end{array}$ & $\frac{000(\mathrm{n}=195)}{\mathrm{AU}(\mathrm{n}=56)}$ & $\mathrm{M} 1(\mathrm{n}=41)$ & $\mathrm{M} 2+(\mathrm{n}=14)$ \\
\hline $\mathbf{1}$ & $\mathrm{B} 1(\mathrm{n}=76)$ & & $\mathrm{M} \times \mathrm{B}(\mathrm{n}=37)$ \\
\hline $\mathbf{2}$ & $\mathrm{B} 2(\mathrm{n}=37)$ & & \\
\hline $\mathbf{3}$ et plus & $\mathrm{B} 3+(\mathrm{n}=22)$ & \\
\hline
\end{tabular}


Figure 4. Evolution de la production laitière au début de la lactation selon les troubles survenus au cours des 2 ème, $3^{e}$ et $4^{e}$ semaines.

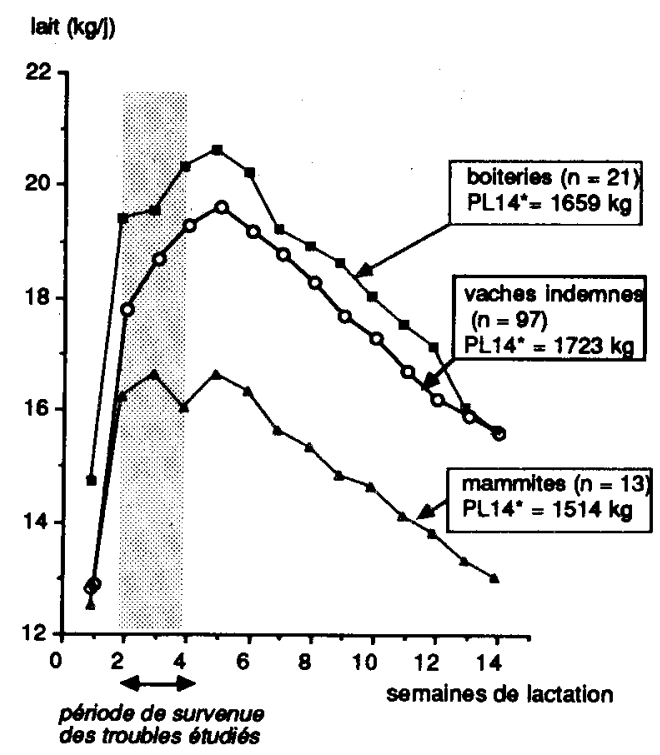

\section{2 / Relations entre les troubles pathologiques et la production laitière}

Les interrelations entre la pathologie et la productivité des vaches laitières ont été successivement analysées à deux échelles, avec des méthodes très différentes:

- à court terme, en comparant sur une période de quelques semaines l'évolution de la courbe de production laitière des vaches atteintes par une affection clinique à celle des vaches indemnes;

- à moyen terme, c'est-à-dire à l'échelle globale de la lactation, en comparant les performances de production selon les différents profils pathologiques de lactation.

\section{a / Influence à court terme des différents types de troubles pathologiques}

\section{Méthode :}

L'évaluation des effets à court terme des troubles sanitaires sur la production laitière a été faite :

1) En début de lactation, en comparant chez les animaux ayant au moins 14 semaines de lactation hivernale, l'évolution au cours de cette période de la production des animaux atteints de mammites $(n=13)$ ou de boiteries $(n=21)$ au cours des semaines 2 à 4 de lactation, à celle des animaux indemnes (profil pathologique $000 ; \mathbf{n}=97)$. Nous n'avons pas retenu dans cette comparaison les lactations ayant présenté des troubles au cours de la première semaine de lactation de façon à disposer d'un indicateur aussi fiable que possible du potentiel laitier (PI = production moyenne des 4ème, 5ème et 6ème jours de lactation (Hoden 1978 et Faverdin et al 1987)).
2) En milieu de lactation (après la $4^{\text {ème }}$ semaine), en comparant l'évolution de la production laitière entre les semaines -3 et +5 par rapport à la semaine où le trouble a été décelé, à l'évolution de la production des animaux indemnes, au même stade de lactation. Cette comparaison a été réalisée pour 5 types de troubles : les boiteries hivernales $(n=59)$, les mammites hivernales $(n=53)$, les boiteries au cours des 2 premières semaines de pâturage $[\mathbf{n}=$ 114 ), les boiteries estivales $(n=24)$ et les mammites estivales $(n=18)$. De plus, pour les 3 premiers types, qui sont les plus représentés, on a étudié l'effet de la race et du numéro de lactation (primipares vs multipares). Les pertes de lait produit relatives à chaque type de trouble ont été calculées par différence entre la courbe de lactation des animaux indemnes et celles des animaux atteints.

\section{Résultats :}

\section{1) Début de lactation :}

L'apparition d'une mammite ou d'une boiterie au cours des semaines 2 à 4 de lactation a entraîné une diminution significative de la production laitière cumulée des 14 premières semaines de lactation de respectivement 209 $(12 \%)$ et $64 \mathrm{~kg}(4 \%)$. Cette diminution est davantage due à une moindre augmentation de la production au cours des premières semaines de lactation, en particulier pour les mammites, qu'à une persistance inférieure après la 5ème semaine (figure 4). Si l'on tient compte du lait non commercialisable (respectivement 84 et 98 $\mathrm{kg}$ ), les pertes totales atteignent 293 et $162 \mathrm{~kg}$ pour les mammites et les boiteries. Cependant, cette estimation pèche largement par défaut, puisque l'allure des courbes montre clairement qu'il n'y a pas de rattrapage à la fin de la période étudiée. Les pertes doivent être largement supérieures à l'échelle de la lactation complète. Malheureusement, notre échantillon ne nous permet pas de le vérifier, en raison de l'insuffisance des effectifs et surtout des perturbations dues aux troubles pathologiques survenant ultérieurement. Les animaux atteints de boiterie en début de lactation présentaient d'autre part un potentiel de production supérieur aux autres : leur production initiale était en effet supérieure de plus de $2 \mathrm{~kg} / \mathrm{j}(\mathrm{P}<0,01)$ à celle des animaux indemnes ou atteints de mammites.

\section{2) Milieu de lactation :}

Les pertes de production entraînées par les troubles pathologiques survenant en milieu de lactation sont en moyenne relativement faibles dans les conditions de l'expérimentation : 0 à $56 \mathrm{~kg}$ (figure 5). Ceci s'explique par la rapidité de la détection et du traitement de ces troubles.

Pour un premier groupe de troubles - boiteries hivernales, boiteries et mammites estivales - la perte de lait produit est pratiquement nulle, et la perte économique varie entre 70 et $85 \mathrm{~kg}$ de lait environ. Les pertes de production sont un peu plus élevées chez les vaches PieNoires que chez les vaches Montbéliardes, et chez les multipares que chez les primipares, sans doute en raison de leur niveau de production supérieur. L'effet de ces troubles est d'autre part très limité dans le temps (figure 5). Les 
Figure 5. Evolution de la production laitière entre les semaines - 3 et +5 par rapport à la détection de divers troubles pathologiques survenant en milieu de lactation.

\section{lait}

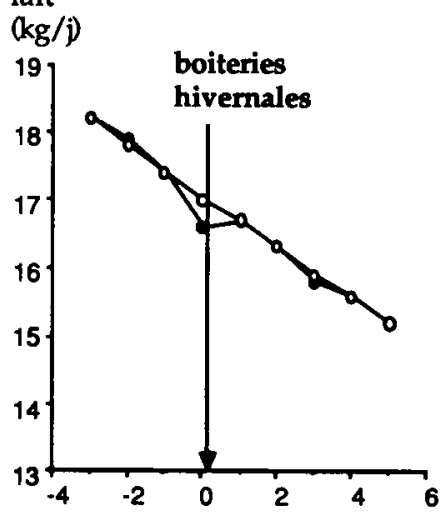

lait

$(\mathbf{k g} / \mathbf{j})$

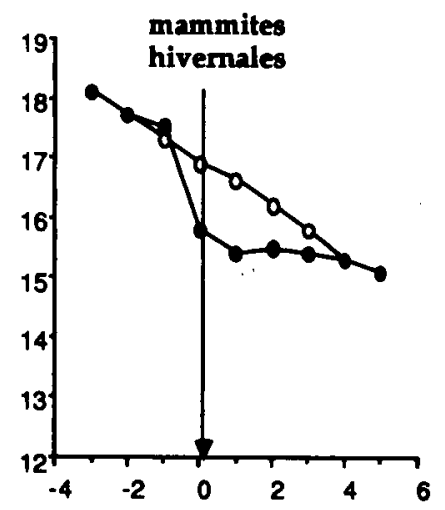

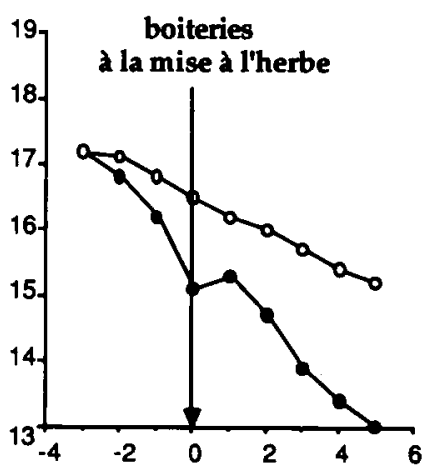

semaines par rapport à la détection du trouble

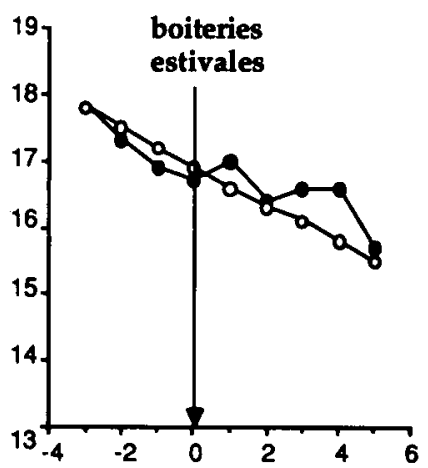

\section{.}

(1)

\section{$O$ animaux indemnes} un trouble pathologique animaux ayant présenté

Les boiteries survenant à la mise à l'herbe entraînent les chutes de production les plus importantes. Cinq semaines après la détection du trouble la production de lait est encore nettement inférieure à celle des animaux indemnes.

semaines par rapport à la détection du trouble

mammites hivernales entraînent des pertes de production plus importantes $(24 \mathrm{~kg}$ en moyenne), en particulier chez les primipares (41 kg contre 18 chez les multipares). La perte économique est de l'ordre de $100 \mathrm{~kg}$ de lait. Les boiteries survenues à la mise à l'herbe entraînent les pertes de production les plus importantes $(56 \mathrm{~kg})$, surtout chez les vaches PieNoires (60 kg contre 11 chez les Montbéliardes) et chez les multipares ( $62 \mathrm{~kg}$ contre $19 \mathrm{chez}$ les primipares). La perte totale atteint en moyenne $133 \mathrm{~kg}$ de lait. Contrairement aux troubles précédents, ces boiteries semblent avoir un effet rémanent important sur la production laitière, puisque l'écart avec la production des animaux indemnes est encore très élevé 5 semaines après la date du trouble, ce qui indique que notre évaluation minimise fortement les pertes réelles.

\section{b / Interrelations entre les profils pathologiques de lactation et les facteurs et composantes de la production laitière totale}

Profils pathologiques

et facteurs expérimentaux

La répartition des lactations entre les divers profils pathologiques définis plus haut est significativement affectée par les facteurs sui- vants (par ordre d'importance décroissant) : année, race et régime alimentaire. Les lactations indemnes de toute atteinte pathologique (type 000) sont beaucoup plus fréquentes en année 5 et 6 ; les types $\mathrm{M} 2+, \mathrm{B} 3+$ et $\mathrm{MxB}$ sont rares durant ces mêmes années. Les vaches Frisonnes sont fortement sur-représentées dans les types B2 et B3+, les Montbéliardes dans le type 000. Aucune relation n'a pu être mise en évidence entre le niveau du concentré et le type de profil pathologique. En revanche, la nature de la ration de base affecte significativement la distribution des profils pathologiques, le profil B3+, s'avérant en particulier, nettement lié aux lots recevant de l'ensilage d'herbe. D'une façon générale, les animaux du lot $\mathrm{MB}$ (Mixte bas) manifestent un comportement pathologique s'apparentant plutôt à celui des animaux des lots $\mathrm{E}$ (Ensilage).

\section{Profils pathologiques et composantes} de la production laitière

Le tableau 3 présente les valeurs moyennes des composantes de la production laitière, par type de profil pathologique. Il apparaît clairement que la production initiale est plus élevée dans les profils $\mathrm{M} 2+, \mathrm{B} 3+$ et $\mathrm{MxB}$ que dans les profils caractérisés par une seule atteinte pathologique (M1, B1, AU) ou indemnes (000). La différence entre la production maximale et 
Tableau 3. Valeurs moyennes des composants de la production laitière, pertes de production, taux de réforme pour raison sanitaire et gravité, par type de profil pathologique de lactation.

\begin{tabular}{|c|c|c|c|c|c|c|c|c|c|}
\hline \multirow{2}{*}{ Composant } & \multicolumn{8}{|c|}{ Type de profil pathologique } & \multirow{2}{*}{ Signification } \\
\hline & 000 & AU & M1 & M2+ & B1 & B2 & B3+ & $\mathbf{M} \times \mathbf{B}$ & \\
\hline Production initiale $(\mathrm{kg} / \mathrm{j})$ & 15,9 & 15,3 & 15,9 & 17,8 & 15,6 & 17,1 & 17,5 & 17,0 & * \\
\hline Production maximale $(\mathrm{kg} / \mathrm{j})$ & 21,1 & 20,7 & 20,7 & 22,4 & 20,7 & 22,6 & 23,3 & 21,7 & ns \\
\hline Durée de lactation (j) & 293 & 272 & 289 & 299 & 292 & 276 & 240 & 301 & ** \\
\hline $\begin{array}{l}\text { Production totale } \\
\text { corrigée }(1)(\mathrm{kg})\end{array}$ & 4181 & 3883 & 3961 & 3955 & 4096 & 3843 & 3479 & 4091 & * \\
\hline $\begin{array}{l}\text { Ecart de production } \\
\text { par rapport au témoin }(2)(\mathrm{kg})\end{array}$ & - & -298 & -154 & -160 & -19 & -272 & -636 & -24 & * \\
\hline $\begin{array}{l}\text { Taux de réforme } \\
\text { pour raisons sanitaires (\%) }\end{array}$ & 0 & 11 & 7 & 11 & 6 & 26 & 50 & 6 & ** \\
\hline $\begin{array}{l}\text { Gravité } \\
\text { (classement) }\end{array}$ & $\begin{array}{c}\text { indemne } \\
(8)\end{array}$ & $\begin{array}{c}\text { pertes } \\
\text { élevées } \\
\text { (3) }\end{array}$ & $\begin{array}{c}\text { pertes } \\
\text { moyennes } \\
(5)\end{array}$ & $\begin{array}{c}\text { pertes } \\
\text { moyennes } \\
(4)\end{array}$ & $\begin{array}{c}\text { pertes } \\
\text { faibles } \\
(7)\end{array}$ & $\begin{array}{c}\text { pertes } \\
\text { élevées } \\
(2)\end{array}$ & $\begin{array}{c}\text { pertes } \\
\text { très } \\
\text { élevées } \\
(1)\end{array}$ & $\begin{array}{c}\text { pertes } \\
\text { faibles } \\
(6)\end{array}$ & \\
\hline
\end{tabular}

(1) Ajustement par analyse de variance incluant les facteurs lot, type génétique et rang de lactation et la covariable production initiale.

(2) Non compris les pertes dues à la non-commercialisation. Pour tous les profils, le témoin est une moyenne pondérée des profils 000 et $\mathrm{AU}$ ( $=4115 \mathrm{~kg}$ ), sauf pour le profil AU où le témoin est le profil 000.

Figure 6. Evolution moyenne de la production laitière selon différents types de profils pathologiques de lactation.
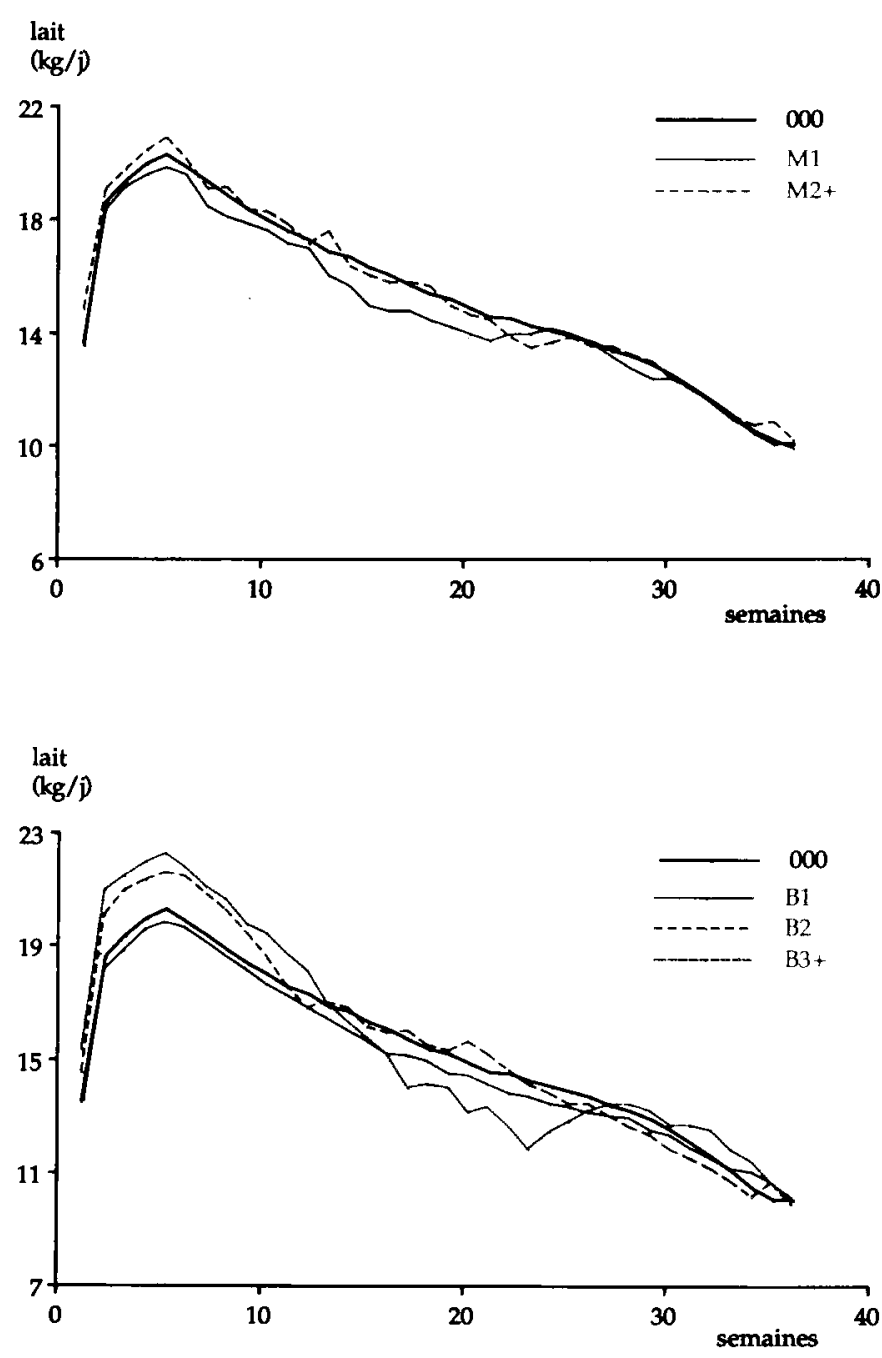

la production initiale est supérieure à $5 \mathrm{~kg}$ dans tous les types de profils, sauf dans ceux avec mammites (M1, M2+, MxB). La durée de lactation n'est sensiblement diminuée que dans le profil $\mathrm{B} 3{ }^{+}$, en raison des réformes survenant en cours de lactation. La production totale moyenne, corrigée pour tenir compte de l'effet des facteurs expérimentaux et du potentiel des animaux (production initiale) est inférieure de près de $300 \mathrm{~kg}$ à celle du témoin dans les profils AU et B2+ et de plus de $600 \mathrm{~kg}$ dans le profil $\mathrm{B} 3+$. Les pertes liées aux mammites (profils $\mathrm{M} 1$ et $\mathrm{M} 2+$ ) sont relativement faibles $(\mathbf{1 6 0} \mathrm{kg})$. Dans ces profils, les pertes surviennent essentiellement dans la première partie de la lactation, et la persistance de la production laitière n'en est pas affectée (figure 6). A l'inverse, les pertes dans les profils à boiteries surviennent en milieu de lactation, au pâturage, et affectent fortement la persistance de la production. Le profil AU est caractérisé par des pertes réparties sur l'ensemble de la lactation.

Quels que soient les indicateurs retenus, les performances de production enregistrées dans le profil $\mathrm{MxB}$ sont étonnantes : les pertes sont en effet très faibles ( $24 \mathrm{~kg}$ ), du même ordre que celles du profil B1 (19 kg), et nettement inférieures à celles du profil M1 (154 kg). Ces résultats sont confirmés par la régularité de la courbe de production de ce profil. Il nous a été totalement impossible d'expliquer cette constatation paradoxale, probablement liée à une pathologie mammaire d'étiologie particulière, et remarquablement bénigne.

\section{c / Caractérisation multifactorielle et typologie des lactations}

Pour cette étude, nous avons réalisé une analyse factorielle des correspondances (AFC) sur l'ensemble des lactations disponibles, en utilisant comme variables actives à la fois certains facteurs fixes (type génétique, rang de lactation), certaines composantes de la production 
180 / J.B. COULON, E. LANDAIS, J.P. GAREL,

Tableau 4. Caractéristiques des différentes classes de lactation selon les résultats de la CAH (répartition en \% sauf pour les 3 dernières variables).

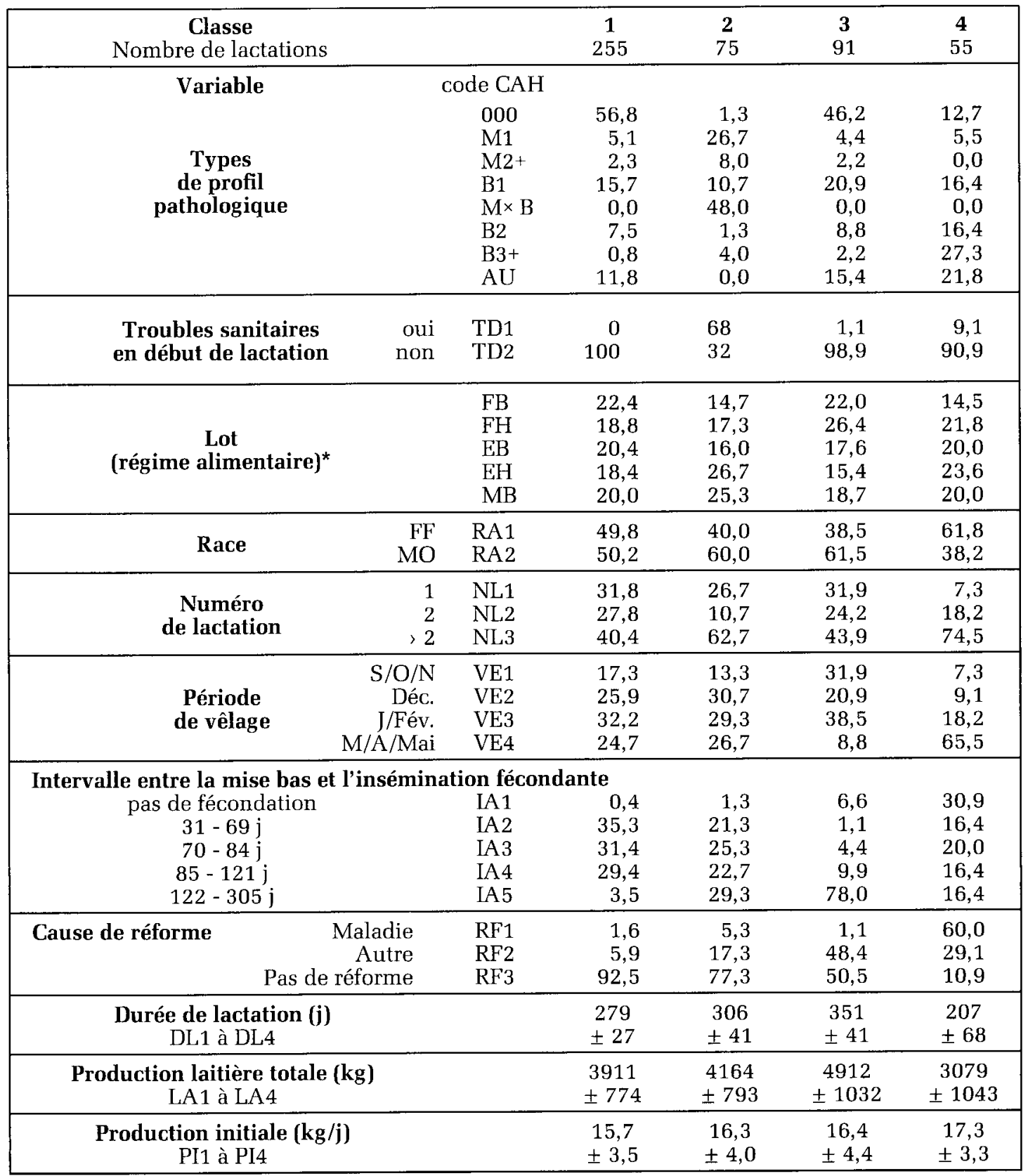

${ }^{*}$ Variable supplémentaire

La classe 1, qui est de beaucoup la plus nombreuse (54\% des lactations) pourrait être définie comme celle des lactations sans problème : elle est associée au profil indemne (000 et TD2), à des intervalles vêlage-insémination fécondante courts (IA 2) et à l'absence de réforme (RF3). La tendance est à la sur-représentation dans cette classe des lactations de rang 1 ou 2 , indépendamment du type génétique. La production initiale moyenne de cette classe est la plus faible, mais le potentiel s'exprime correctement. En ce qui concerne les régimes hivernaux, la tendance est légèrement en faveur des bas niveaux d'alimentation (lots B).

La classe 2 est principalement définie par la présence de mammites (association forte avec
M1, M2 et surtout MxB, association avec TD1). Il s'agit plutôt de lactations de vaches Montbéliardes âgées. La production initiale est moyenne $(16,3 \mathrm{~kg}$ ), mais le potentiel s'exprime correctement, comme le montre la figure 8 . Sur le plan alimentaire, la tendance est à l'association de cette classe avec les lots EH et MB.

La classe 3 est définie à la fois par sa productivité élevée (4 $912 \mathrm{~kg}$ en moyenne, soit près de $700 \mathrm{~kg}$ de plus que la précédente, pour une production initiale équivalente) et par l'importance des problèmes de reproduction : intervalles vêlage-insémination fécondante longs (supérieurs à 120 jours dans près de $80 \%$ des cas) et près de $50 \%$ de réformes liées à la reproduction. La durée de lactation est logiquement élevée, mais la figure 8 montre clairement 
que ce n'est pas la principale raison de l'accroissement de la production totale : cette classe est en effet caractérisée par une persistance élevée de la production laitière. Ceci s'explique en partie par la prédominance de vêlages d'automne, d'animaux de race Montbéliarde et de primipares. En ce qui concerne l'alimentation, cette classe est plutôt associée aux régimes à base de foin (lots F).

La classe 4 est caractérisée par la présence de troubles pathologiques graves (boiteries répétées, "autres troubles") et par l'infécondité (IA1), entraînant la réforme dans 9 cas sur 10. Cette classe de lactations est fortement liée à des facteurs généralement associés à une forte production laitière : race Frisonne, rang de lactation élevé, production initiale maximum, vêlages décalés en fin de saison. Cependant, en raison des troubles pathologiques enregistrés, la durée de lactation et surtout la persistance (figure 8) sont très affectées, et la production laitière totale est finalement la plus faible des quatre classes ( $3079 \mathrm{~kg}$ en moyenne). Il est à noter que le triptyque vêlage tardif/boiteries au pâturage/réforme constitue sur le domaine de Marcenat un tableau très caractéristique de la fin de carrière des vaches Frisonnes, et que les lactations correspondantes appartiennent à la classe 4 de la CAH.

\section{3 / Etude à l'échelle de la carrière}

\section{1 / Analyse de la longévité}

Cette étude porte sur 136 carrières de vaches laitières, étudiées à partir de leur premier vêlage. Sur ce total, seules 87 carrières ont été suivies jusqu'à la réforme des animaux. Les 49 autres se poursuivaient lorsque l'expérimentation a été interrompue, après six années d'observation. De ce fait, l'échantillon des carrières complètes sélectionne les carrières les plus courtes, et les données ont dû être corrigées pour éliminer ce biais (Coulon et al 1989b). Cette méthode a permis d'analyser les effets sur
Figure 8. Evolution moyenne de la production laitière des 4 classes de lactation de la typologie.

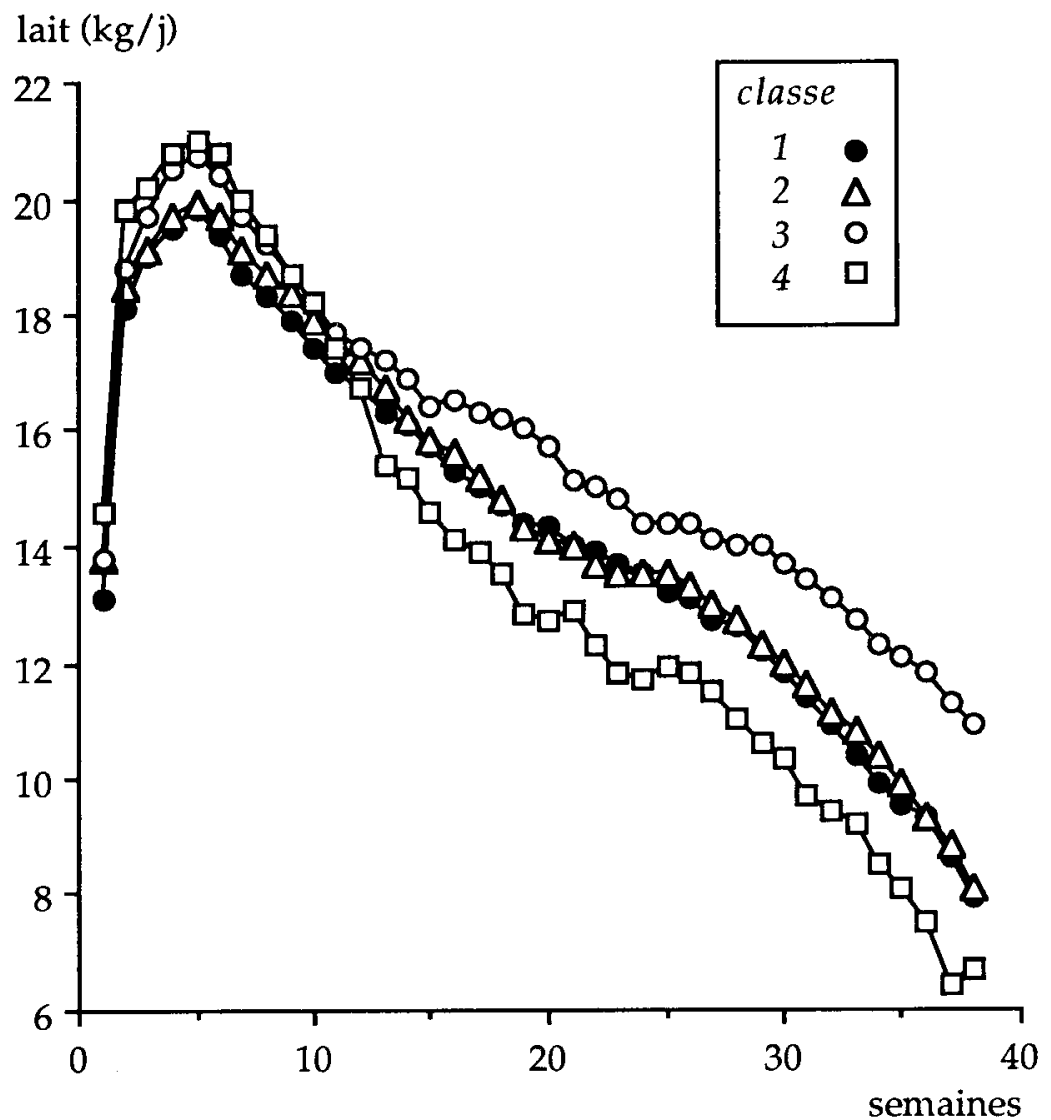

la longévité des facteurs fixes suivants : la race, le régime alimentaire et le profil pathologique de la première lactation (tableau 5).

La race n'a pas eu d'effet significatif sur la durée moyenne des carrières (respectivement 3,29 et 3,31 lactations pour les Pie-Noires et les Montbéliardes). Cependant les profils de carrière sont distincts d'une race à l'autre, en rai-

Tableau 5. Variations de la longévité moyenne des vaches selon la race, le régime alimentaire hivernal et le profil pathologique de la première lactation.

\begin{tabular}{|ccccc|}
\hline Facteur & & Effectif & $\begin{array}{c}\text { Longévité moyenne } \\
\text { (nombre de lactations) } \\
\text { et signification (1) }\end{array}$ \\
\hline Race & FF & 65 & 3,29 & \\
& MO & 71 & 3,31 & $\mathrm{~ns}$ \\
\hline Régime & $\mathrm{FB}$ & 26 & 3,18 & $\mathrm{bc}$ \\
alimentaire & $\mathrm{FH}$ & 29 & 3,25 & $\mathrm{bc}$ \\
hivernal & $\mathrm{EB}$ & 28 & 2,50 & $\mathrm{a}$ \\
(code du lot) & $\mathrm{EH}$ & 27 & 3,47 & $\mathrm{c}$ \\
& $\mathrm{MB}$ & 25 & 3,02 & $\mathrm{~b}$ \\
\hline & 000 & 60 & 3,43 & $\mathrm{~d}$ \\
pathologique & $\mathrm{M} 1 / \mathrm{M} 2+$ & 14 & 2,80 & $\mathrm{~b}$ \\
en première & $\mathrm{B} 1$ & 22 & 3,12 & $\mathrm{c}$ \\
lactation & $\mathrm{M} \times \mathrm{B}$ & 10 & 3,22 & $\mathrm{~cd}$ \\
& $\mathrm{~B} 2 / \mathrm{B} 3+$ & 12 & 2,31 & $\mathrm{a}$ \\
& $\mathrm{AU}$ & 18 & 2,62 & $\mathrm{~b}$ \\
\hline
\end{tabular}

(1) Les valeurs suivies de lettres différentes sont significativement différentes $(\mathrm{P}<0,05)$. 
Figure 9. Effet de la race sur la longévité des vaches (pourcentage de vaches encore présentes selon le rang de lactation).

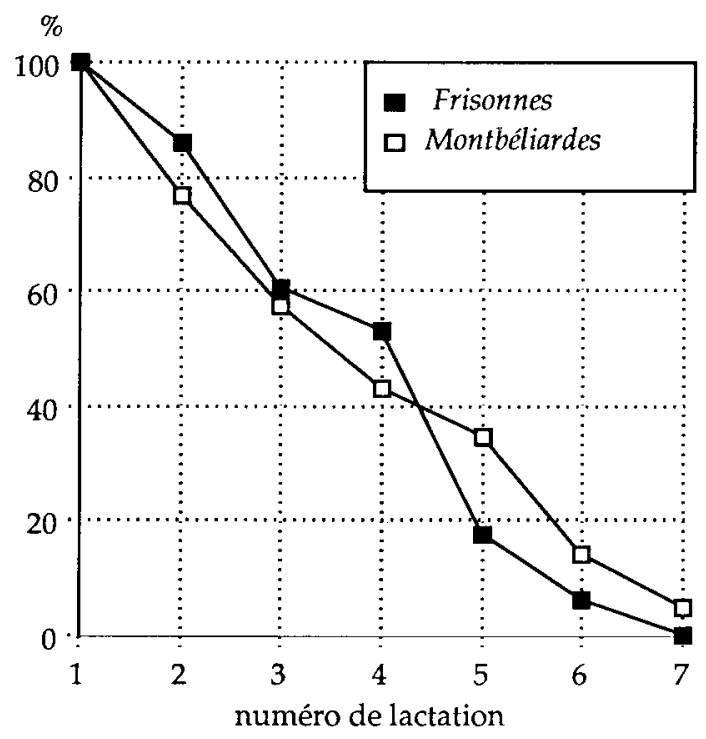

son de taux de réforme différents, notamment à l'issue de la première lactation $(23 \%$ pour les Montbéliardes contre $14 \%$ pour les PieNoires). Après une sélection plus sévère en début de carrière, principalement pour des raisons relatives à leurs performances, les vaches Montbéliardes manifestent donc une longévité supérieure à celle des Pie-Noires (figure 9).

Le régime alimentaire entraîne des différences significatives de longévité. Les lots à faible niveau d'alimentation recevant de l'ensilage

Figure 10. Enchaînement des profils pathologiques de lactation entre 2 lactations consécutives. Les profils doublés de rouge correspondent à une aggravation de l'état sanitaire entre la lactation $n$ et la lactation $n+1$. Ceux doublés de blanc correspondent à une amélioration de cet état. d'herbe (EB et, dans une moindre mesure, $\mathrm{MB}$ ) ont la longévité la plus faible (respectivement 2,50 et 3,02 lactations) et ceux à haut niveau d'alimentation (FH et $\mathrm{EH}$ ) la longévité la plus élevée (respectivement 3,25 et 3,47 lactations).

Le profil pathologique de la première lactation entraîne également des variations significatives de la longévité. Les vaches indemnes (000) ou atteintes des troubles les plus bénins $(\mathrm{MxB}$ et $\mathrm{B} 1)$ au cours de leur première lactation présentent une longévité supérieure de 0,6 lactation à celles atteintes de troubles graves (M1/ $\mathrm{M} 2+$ et $\mathrm{AU}$ ) et de 1 lactation à celles atteintes de troubles très graves $(\mathrm{B} 2 / \mathrm{B} 3+)$. Cette observation met en évidence l'importance de la première lactation pour la carrière ultérieure des vaches.

\section{2 / Enchaînement des profils pathologiques de lactation au cours de la carrière}

Pour cette étude, nous avons analysé sous l'angle pathologique l'enchainement des lactations au cours d'une même carrière (analyse longitudinale) et, dans un premier temps, les séquences de deux lactations consécutives, Ln et $\mathrm{Ln}+1$, quel que soit leur rang. La figure 10 résume les résultats de cette étude. Elle a été dressée en tenant compte de la gravité des troubles de sorte que les profils pathologiques doublés de rouge correspondent à une aggravation de l'état sanitaire de l'animal considéré entre la lactation $n$ et la lactation $n+1$, et ceux doublés de blanc, à l'inverse, à une amélioration de cet état. Le type 000, qui se succède à lui-même dans $41 \%$ des cas, est de beaucoup le plus stable, suivi par le type AU (21 \%) et le type M1/ $\mathrm{M} 2+(14 \%)$. Les types caractérisés par la présence de boiteries (B1, B2/B3+ et même $\mathrm{M} \times \mathrm{B}$ ) sont très instables, ce qui ne s'explique que partiellement par le jeu des réformes. Ainsi, les boiteries, particulièrement récidivantes à
Répartition des profils pathologiques en lactation $n+1$
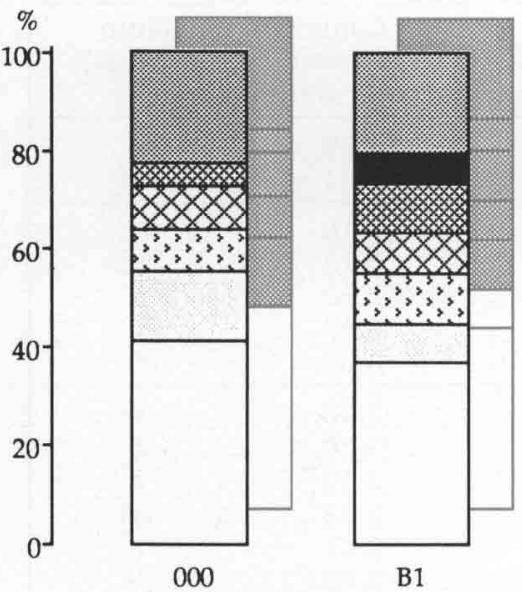

B1

\begin{tabular}{|lll}
$\square$ 000 & AU & Réformes maladies \\
$\square$ B1 et M*B & B2/B3+ & Réformes autres \\
M1/M2 $_{3}^{3}$ & & \\
\hline
\end{tabular}
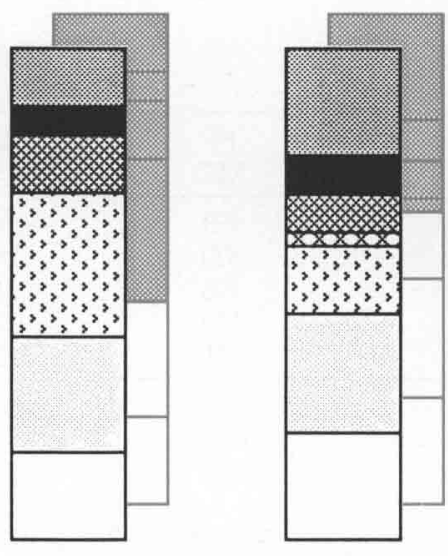

M1/M2+

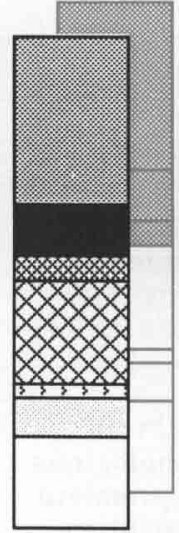

$\mathrm{AU}$

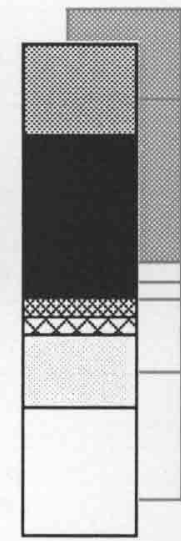

B2/B3+

Profil pathologique de la lactation $n$ 
l'échelle d'une lactation n'apparaissent pas comme des troubles susceptibles de caractériser une carrière. Plus généralement, la distribution observée est très dispersée: un profil donné peut pratiquement suivre ou précéder n'importe quel autre profil. De ce fait, il s'avère très difficile de caractériser les carrières des vaches laitières exclusivement à partir de la séquence des profils pathologiques de lactation. L'étude des carrières comprenant au moins trois lactations consécutives a confirmé cette observation. Cette caractérisation doit donc être recherchée à partir de facteurs multiples.

\section{3 / Enchaînement des classes de lactation au cours de la carrière}

L'analyse a été abordée, comme précédemment, par l'étude des séquences de deux lactations (figure 11). Seule la classe 1 présente une certaine stabilité d'une lactation sur l'autre, se succédant à elle-même dans $51 \%$ des cas. Les trois autres classes sont en revanche remarquablement instables. La classe 4, qui débouche dans $97 \%$ des cas sur une réforme, est clairement une classe de fin de carrière. La classe 3 (qui regroupe de nombreuses lactation de rang 1) débouche principalement sur des réformes liées aux problèmes de reproduction et sur des lactations de classe 1 . La classe 2 (caractérisée par la présence de mammites), bien que regroupant de nombreuses lactations de vaches âgées, débouche sur un faible taux de réforme (17\%), et dans plus de $40 \%$ des cas sur des lactations de classe 1.

La distribution des différentes classes reste globalement stable d'une lactation sur l'autre, sous l'effet de la politique de réforme, qui contrecarre la tendance spontanée à la raréfaction de la classe 1 et à l'accroissement de la fréquence des classes 3 et 4 .

L'étude des carrières de trois lactations et plus montre clairement que les classes 2 et surtout 1 sont les plus favorables à la longévité des vaches: près de $70 \%$ des carrières de plus de deux lactations débutent par une lactation de classe 1 , et $18 \%$ par une lactation de classe 2 . Parmi les carrières de plus de trois lactations, $67 \%$ ont débuté par une lactation de classe 1 et $24 \%$ par une lactation de classe $2 ; 56 \%$ ont débuté par une séquence de type 1-1 et $18 \%$ par une séquence de type 2-1. A l'inverse, les carrières courtes sont caractérisées par la fréquence élevée des lactations des classes 3 et 4 . La classification proposée constitue donc un élément important d'interprétation de la longévité des vaches laitières.

\section{4 / Evolution des performances de production au cours de la carrière}

En moyenne, les 50 vaches ayant réalisé au moins 3 lactations et ayant eu au moins 6 semaines de lactation hivernale à chacune de leur lactation, ont produit $3794 \mathrm{~kg}$ de lait en première lactation et ont augmenté leur production totale de respectivement 352 puis $270 \mathrm{~kg}$ en deuxième et troisième lactation. Leur
Figure 1\%. Enchainement des classes de lactation entre 2 lactations consécutives.
Répartition des classes en lactation $n+1$

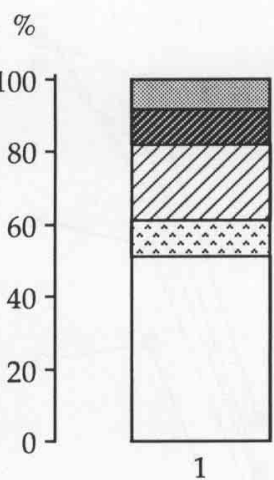

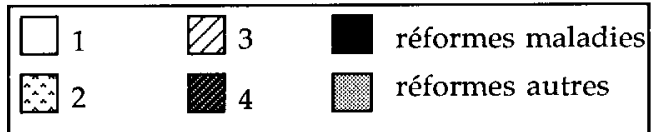
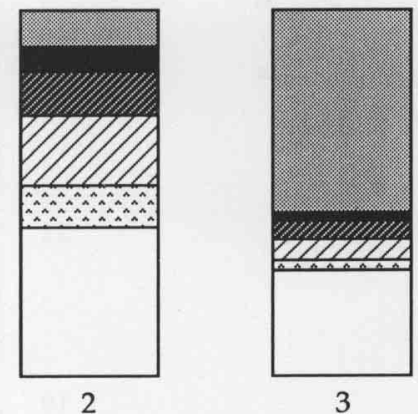

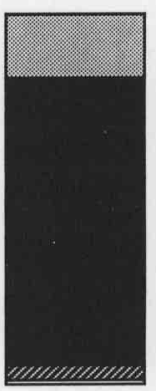

4 classe de la lactation $n$

production laitière au cours des 6 premières semaines de lactation est passée de $15,8 \mathrm{~kg} / \mathrm{j}$ en première lactation à $19,4 \mathrm{~kg} / \mathrm{j}$ en deuxième lactation et à $20,0 \mathrm{~kg} / \mathrm{j}$ en troisième lactation. Leur poids vif au vêlage a augmenté de 24 et de 51 $\mathrm{kg}$ entre la première lactation et les lactations 2 et 3 . Bien que cet échantillon diffère notablement de l'ensemble de la population expérimentale en ce qui concerne les classes de lactation, ces valeurs sont semblables à celles observées sur l'ensemble des animaux (cf Coulon et al 1985); en particulier, la production initiale moyenne des premières lactations des vaches ayant réalisé au moins 3 lactations n'est pas dif-

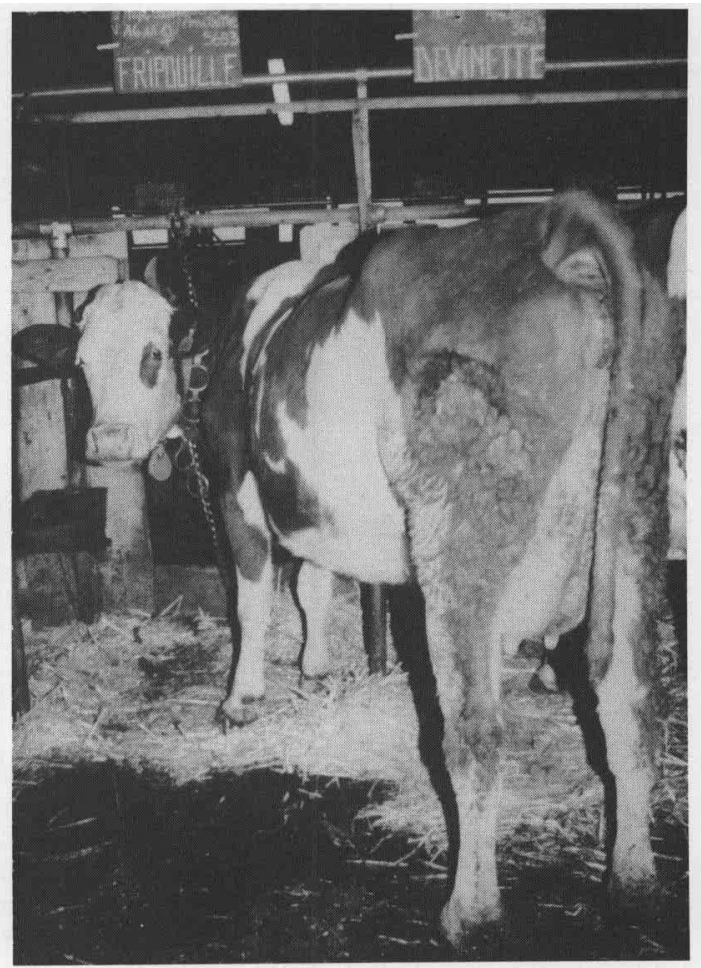

La longue durée de stabulation en zone de montagne peut être un facteur prédisposant aux boiteries. 
Figure 12. Evolution des variables de production laitière de la première à la troisième lactation.
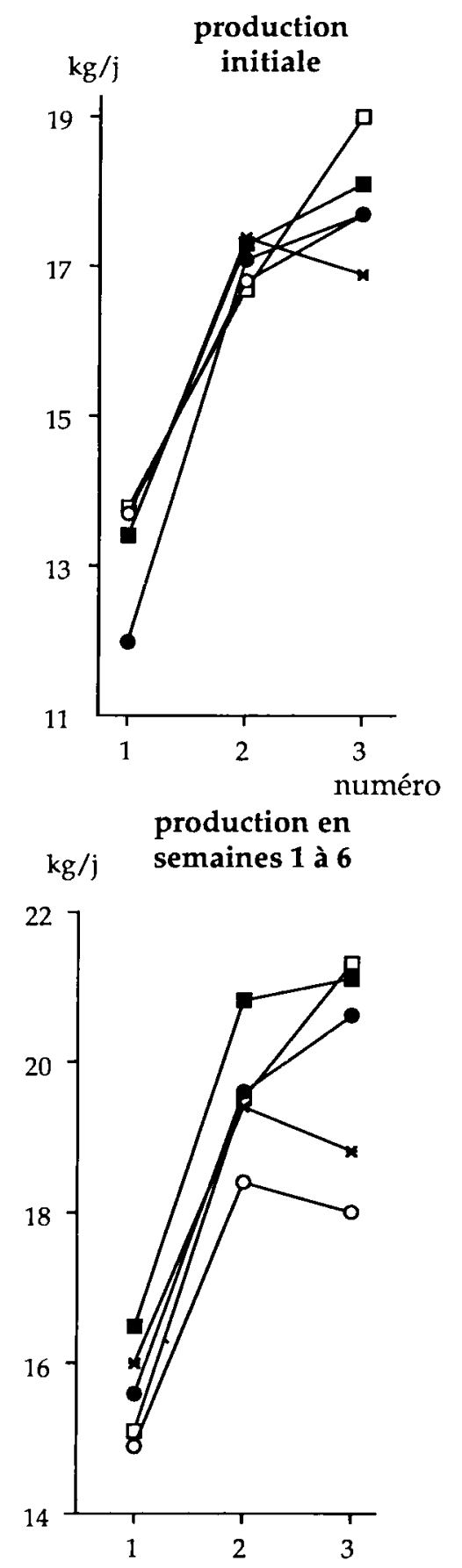

numéro de lactation

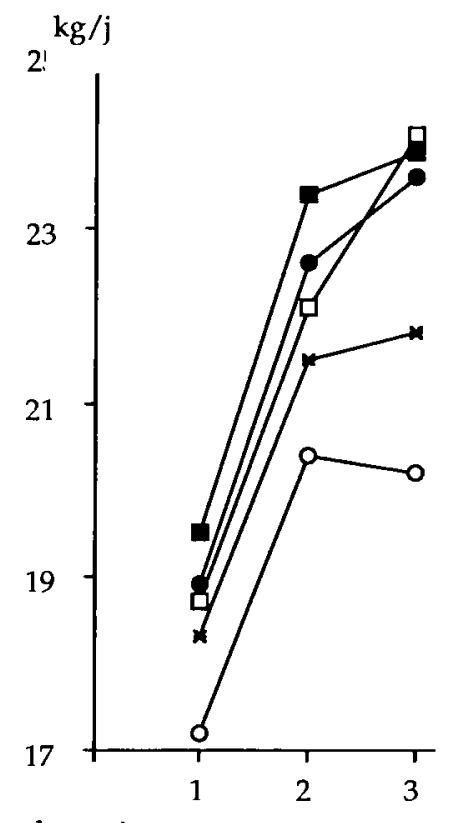

de lactation
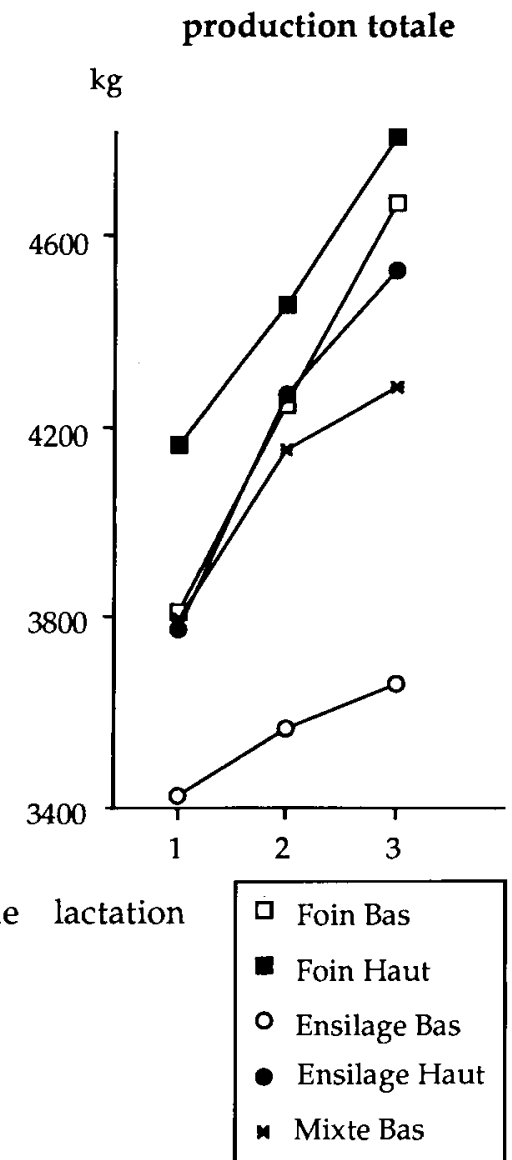

férente de celle de l'ensemble des primipares (respectivement 13,3 et $12,9 \mathrm{~kg} / \mathrm{j}$ ). Les réformes ont donc touché aussi bien des vaches fortes productrices (pour des raisons liées à leur reproduction ou à la pathologie) que des vaches faibles productrices (pour des raisons liées précisément à leur niveau de production insuffisant, ou à la pathologie).
Les vaches recevant une ration à base de foin ont produit (en moyenne sur les 3 lactations) plus de lait que celles recevant une ration à base d'ensilage d'herbe, aussi bien au cours des 6 premières semaines de lactation $(19,3 \mathrm{~kg} / \mathrm{j}$ contre 17,9 ), qu'au cours de la lactation totale (4 $359 \mathrm{~kg}$ contre 3 871), et ce d'autant plus que leur rang de lactation avançait : l'écart de production laitière est ainsi passé de $387 \mathrm{~kg}$ en première lactation à $646 \mathrm{~kg}$ en troisième lactation (figure 12).

Les vaches recevant un haut niveau d'apport énergétique (lots $H$ ) ont produit, en moyenne sur 3 lactations, $1,1 \mathrm{~kg} / \mathrm{j}$ de lait en plus au cours des 6 premières semaines de lactation et $376 \mathrm{~kg}$ de plus à l'échelle de la lactation totale que les vaches recevant un faible niveau d'apport énergétique (lots B). Mais le résultat le plus frappant à ce niveau concerne l'interaction qui est mise en évidence entre le type de ration de base et le niveau des apports énergétiques : la différence de production entre lots "Haut " et «Bas » est beaucoup plus importante pour les lots «Ensilage" (639 kg par lactation en moyenne) que pour les lots "Foin» $(233 \mathrm{~kg}$ ) (figure 12). Cet effet se retrouve sur diverses composantes de la production, comme la production cumulée des six premières semaines ou la production maximale. Ces écarts sont directement liés aux niveaux d'apport énergétique différents des 2 rations de base, dûs dans cet essai à l'ingestibilité inférieure de l'ensilage. Dans ces conditions, l'efficacité alimentaire marginale de l'aliment concentré est pratiquement deux fois plus importante avec la ration à base d'ensilage d'herbe qu'avec celle à base de foin (respectivement $+1,4$ et $+2,3 \mathrm{~kg}$ de lait en plus par $\mathrm{kg}$ de concentré supplémentaire, en moyenne sur les trois premières lactations).

Au total, on est conduit à regrouper les lots $\mathrm{FB}, \mathrm{FH}$ et $\mathrm{EH}$, qui ont présenté des augmentations de production importantes et régulières entre la première et la troisième lactation (respectivement $+752 \mathrm{~kg},+4,8 \mathrm{~kg} / \mathrm{j}$ et $+5,3 \mathrm{~kg} / \mathrm{j}$ pour les productions totale, maximale et des semaines 1 à 6), et les lots $\mathrm{EB}$ et MB pour lesquels ces augmentations ont été faibles (respectivement $+359 \mathrm{~kg},+3,3 \mathrm{~kg} / \mathrm{j}$ et $+3,0 \mathrm{~kg} / \mathrm{j})$, et même parfois négatives entre la deuxième et la troisième lactation (figure 12). Ces résultats sont confirmés par l'examen des courbes d'évolution de la production laitière de chaque lot (figure 13). Les lots EB et MB se caractérisent ainsi par des courbes d'évolution de la production laitière dont le pic est tronqué en première lactation et par la faible augmentation de production après la deuxième lactation. Le lot FB présente aussi une courbe fortement tronquée en première lactation (en partie compensée au pâturage), mais continue d'augmenter sa production au-delà de la deuxième lactation. Les lots $\mathrm{FH}$ et surtout $\mathrm{EH}$ présentent des évolutions de production très régulières et des persistances hivernales élevées (en première lactation pour le lot $\mathrm{FH}$ en particulier)

En définitive, les traitements hivernaux ont entraîné, à l'échelle des 3 lactations cumulées, des écarts de production très importants, atteignant $2772 \mathrm{~kg}$ entre les lots extrêmes (FH et 


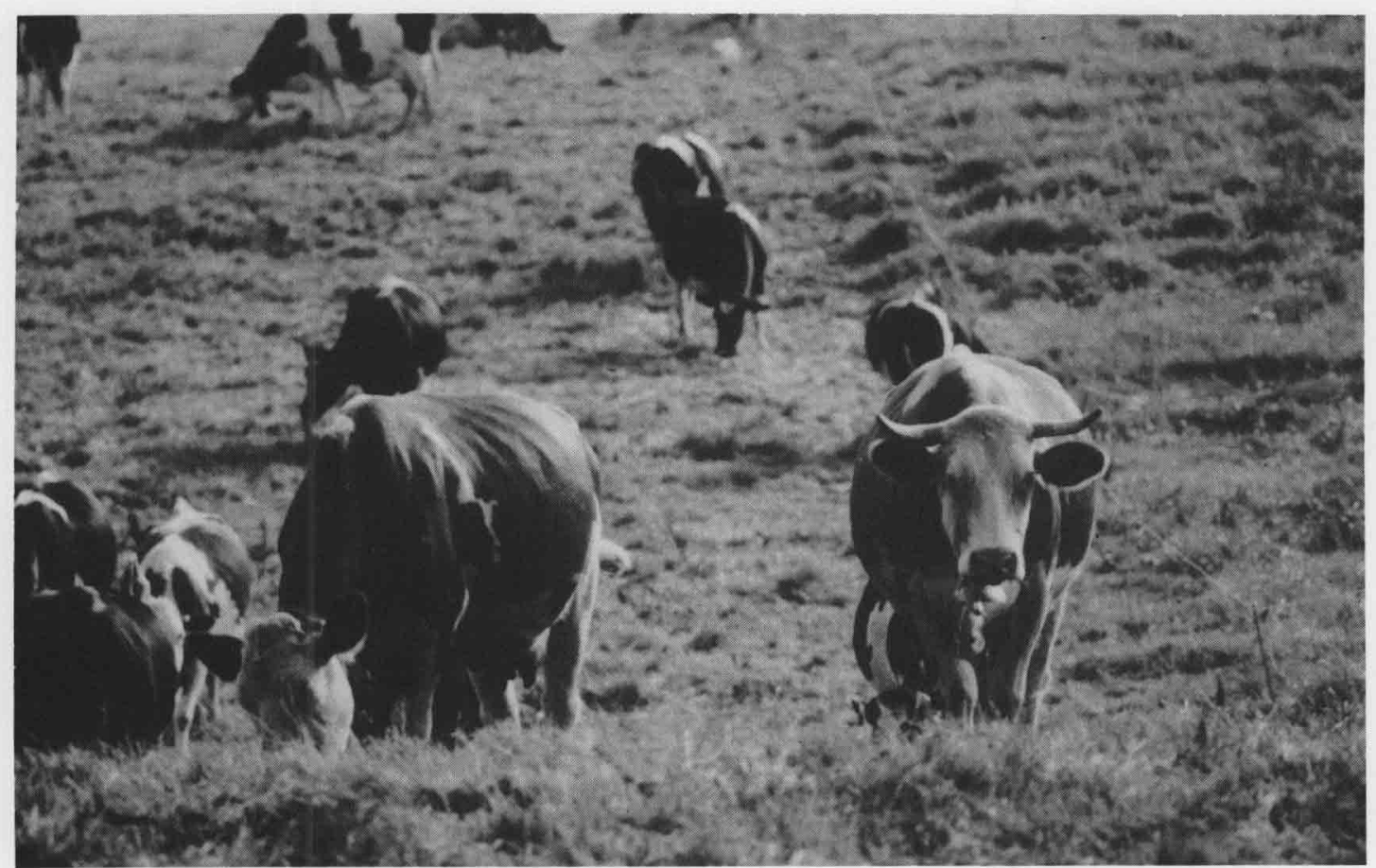

Au pâturage, les gains de poids vif ont été légèrement supérieurs pour les vaches alimentées au niveau bas pendant l'hiver. Au total, les variations de poids entre la première et la troisième lactation ont été similaires pour tous les régimes alimentaires hivernaux.

EB), soit $26 \%$. Cet écart est de $699 \mathrm{~kg} \mathrm{(5 \% )}$ entre les lots FB et $\mathrm{FH}$ et de $1917 \mathrm{~kg}(18 \%)$ entre les lots EB et EH.

Ces résultats sont cependant biaisés en raison

1) des différences de potentiel de production des animaux d'un lot à l'autre, et en particulier du niveau plus faible du lot EH. La prise en compte de cet écart de potentiel accentue les écarts de production à l'échelle des 3 lactations entre les lots $\mathrm{EH}$ et $\mathrm{EB}(2690 \mathrm{~kg}$ ) et montre que les productions des lots $\mathrm{FH}$ et $\mathrm{EH}$ sont identiques (respectivement 13240 et $13070 \mathrm{~kg}$ de lait sur les 3 lactations) ;

2) dans une moindre mesure par les différences de durée de lactation d'un lot à l'autre: le lot EB présente en particulier des lactations plus courtes d'environ 20 jours que celles des autres lots ;

3) enfin, par l'incidence différente des troubles sanitaires sur la production d'un lot à l'autre : en particulier la proportion de lactations atteintes de troubles graves (AU, B2, B3+) est passée de 40 à $10 \%$ entre la première et la troisième lactation dans le lot $\mathrm{FB}$ et à l'inverse de 21 à $37 \%$ dans les lots "Haut". Ceci peut expliquer l'évolution différente de la production laitière d'une lactation à l'autre dans ces lots (figure 12)

L'évolution des performances des vaches Frisonnes et Montbéliardes entre la première et la troisième lactation a été comparable (respectivement $+576 \mathrm{~kg}$ de lait contre $+508 \mathrm{~kg})$, mais plus rapide chez les Frisonnes que chez les Montbéliardes : chez les Frisonnes, l'augmentation de production entre la première et la deuxième lactation a été 2 fois plus importante $(+465 \mathrm{~kg}$ contre $+223 \mathrm{~kg})$, et les augmenta- tions des productions initiale, maximale et des semaines 1 à 6 ne se sont pas poursuivies audelà de la deuxième lactation.

\section{5 / Evolution du poids vif au cours de la carrière}

Les variations de poids vif ont été identiques dans les 2 races quel que soit le numéro de lactation.

Les pertes de poids vif hivernales ont été d'autant plus importantes que le numéro de lactation des vaches était plus élevé (respectivement - 16, - 23 et - $37 \mathrm{~kg}$ pour les lactations de rang 1,2 et 3 ) et que leur niveau d'alimentation était plus faible (-19 $\mathrm{kg}$ pour les lots « Haut» contre $-34 \mathrm{~kg}$ pour les lots «Bas ») (figure 13). A l'inverse, les gains de poids vif estivaux ont été légèrement supérieurs dans les lots "Bas" ( $+97 \mathrm{~kg}$ en 20 semaines de pâturage), comparativement aux lots "Haut» $(+70 \mathrm{~kg})$, de sorte que les variations de poids vif entre la première et la troisième lactation ont été en moyenne peu différentes d'un lot à l'autre $(+35$ à +58 $\mathrm{kg}$ selon les lots). Cette évolution a cependant été très différente entre le lot $\mathrm{EB}$, où les vaches n’ont pas gagné de poids entre la première et la deuxième lactation, et les autres lots où l'augmentation de poids vif s'est réalisée au cours des 2 lactations (respectivement 30 et $25 \mathrm{~kg}$ ) (figure 13). L'interprétation de ce résultat est cependant rendu malaisée par la différence de poids initial entre les vaches de ce lot EB (542 $\mathrm{kg}$ ) et les autres $(510 \mathrm{~kg})$.

Il n'a pas été possible de mettre en évidence de relation entre les évolutions individuelles de poids vif et celles de production laitière d'une lactation à l'autre. 
Figure 3 . Evolutions de la production laitière et du poids vif au cours de la lactation en fonction du lot et du numéro de lactation.
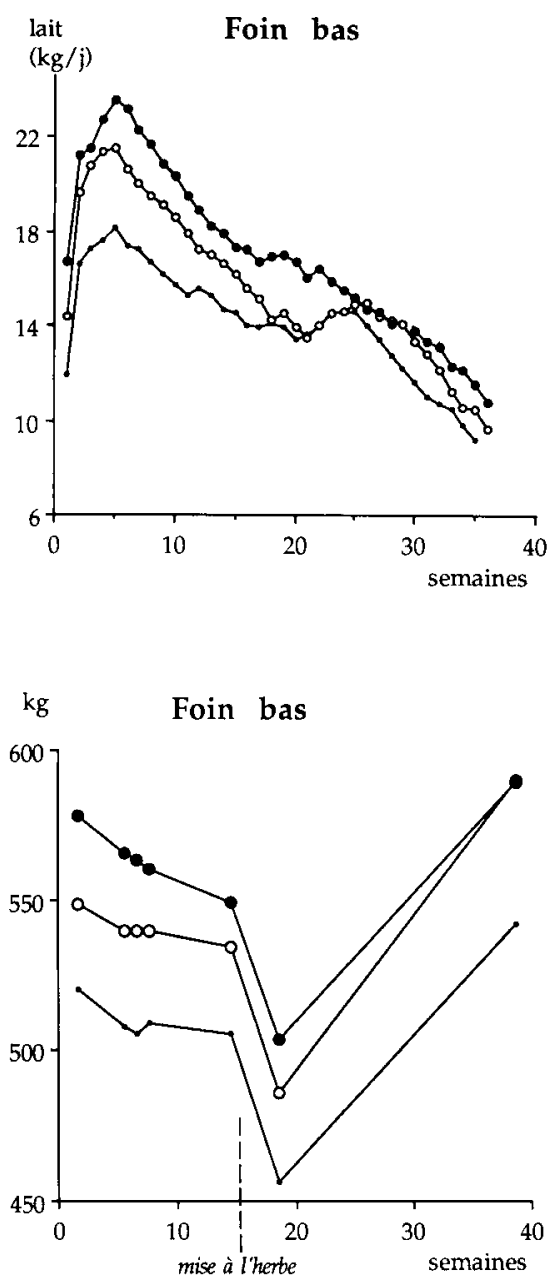
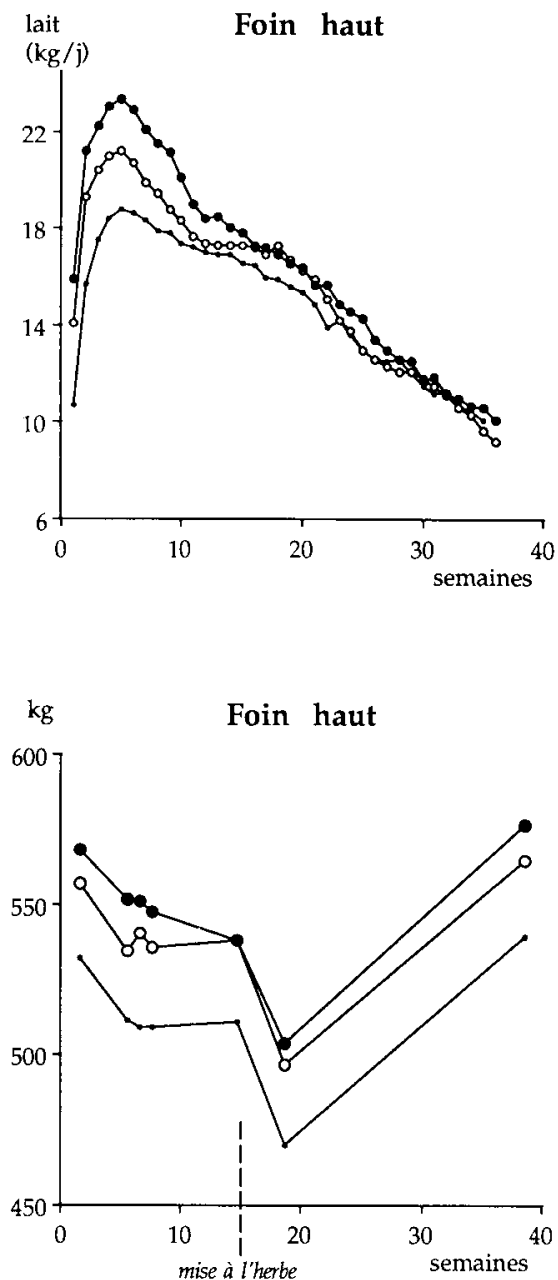

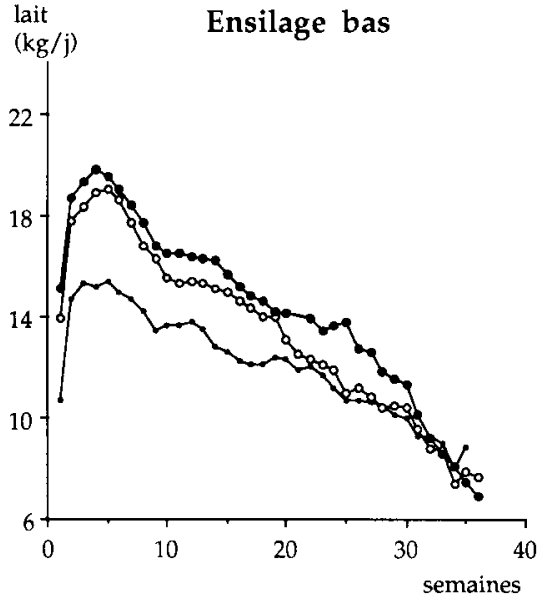

$\mathrm{kg}$

Ensilage bas

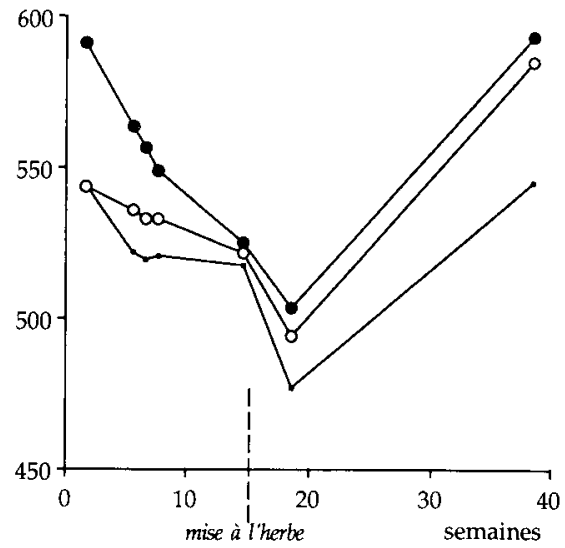

\section{Discussion}

D'une manière générale, le traitement des données de cette étude a posé un certain nom- bre de problèmes liés en partie aux conditions de l'étude (absence de notation de la gravité des troubles sanitaires, des causes des troubles de la reproduction) mais pour la plupart inhérents à la notion même d'essai à long terme (perturbation de l'analyse des carrières par l'évolution des conditions d'environnement, interruption de ces carrières par la fin de l'expérience ...) et à l'approche globale que nous avons voulu adopter (mise en place d'une typologie des lactations).

D'autre part, les résultats quantitatifs de cette étude n'ont qu'une validité limitée au milieu particulier dans lequel ils ont été obtenus. Les comparaisons entre les deux types génétiques en présence (Montbéliard et Pie-Noir) doivent notamment être interprétés en se souvenant qu'il s'agissait de vaches Françaises Frisonnes. Ils ne sont certainement pas extrapolables au type Holstein aujourd'hui largement répandu dans l'élevage français, et qui manifeste en particulier une résistance supérieure vis-à-vis des affections podales. Par contre, les méthodes utilisées (décomposition des fréquences pathologiques brutes en fréquence des lactations atteintes et nombre d'épisodes pathologiques par lactation atteinte; évaluation à court et à moyen terme des troubles sanitaires sur la production laitière; analyse de la longévité des animaux ...) et les phénomènes qui ont été mis en évidence, principalement au niveau des interactions entre alimentation, pathologie, reproduction et production laitière, ont une portée plus générale et constituent une base nouvelle de réflexion et d'analyse des résultats, pour une gamme étendue de situations.

L'originalité de la démarche adoptée tient en partie à sa capacité de mettre efficacement en relation les variables relatives à la production laitière et celles qui rendent compte de la pathologie observée en cours d'essai. Cette mise en relation a rarement été faite jusqu'ici, faute d'une méthode adaptée. Nos résultats montrent cependant toute son importance, puisque les profils pathologiques apparaissent en fin de compte comme les principaux facteurs de variation de la production dans les conditions de notre expérimentation. 

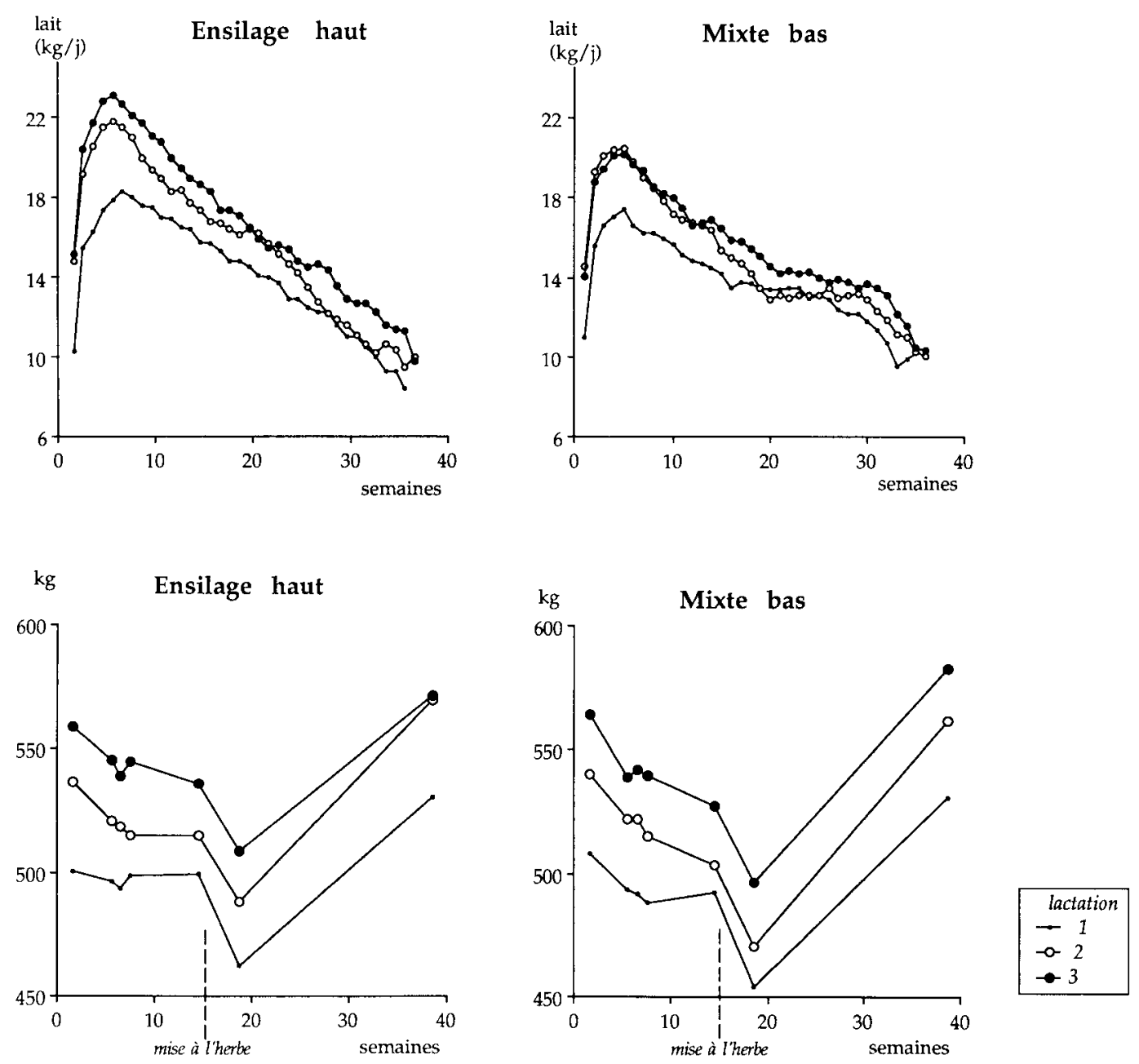

Au-delà de ces remarques, cette étude a permis de mettre en évidence un certain nombre de résultats originaux et spécifiques concernant :

1) la précision des effets propres du stade de lactation et de la saison sur les mammites et les boiteries, et la mise en évidence de l'important effet de relais entre ces 2 troubles au cours de l'année ;

2) l'effet défavorable des rations à base d'ensilage d'herbe sur la fréquence des boiteries et son accentuation au cours de la carrière des animaux. Ce résultat confirme des observations réalisées sur le terrain (Brochart et Fayet 1981, Faye 1989) mais ses causes restent encore à définir :

3) l'importance quantitative des troubles sanitaires à court et à moyen terme sur la production laitière et leur effet variable selon le stade de lactation ou la période de l'année auxquels ils surviennent. Ces résultats précisent ceux obtenus par Marquis et Hoden (1977) et Lucey et Rowlands (1984). A l'échelle de la lactation, ces effets sont parfois très importants (cas des boiteries répétées), et supérieurs à ceux des traitements alimentaires hivernaux. Leur prise en compte dans l'appréciation de la producti vité des animaux est donc primordiale :

4) les effets cumulatifs considérables des traitements alimentaires hivernaux sur la production laitière: l'écart de production qui était en moyenne à l'échelle de la lactation de $160 \mathrm{~kg}$ entre les lots FB, FH et $\mathrm{EH}$ et les lots $\mathrm{EB}$ et $\mathrm{MB}$ (Coulon et al 1985) passe à près de $500 \mathrm{~kg}$ par lactation quand on considère les 3 premières lactations. De plus, cet écart augmente fortement d'une lactation sur l'autre atteignant successivement 305,462 et $698 \mathrm{~kg}$.

5) l'importance des caractéristiques, en particulier sanitaires, de la 1ère lactation sur la longévité des animaux.

L'ensemble de ces résultats conduit à relativiser les conclusions tirées d'une analyse des données réalisée à l'échelle d'une seule lactation et privilégiant la relation régime alimentaire hivernal - production laitière des animaux. Ils conduisent à la notion de typologie de lactations que nous avons présentée et à celle, qu'il faudrait développer, de typologie de carrières.

Ceci revient à traiter les résultats concernant la pathologie, la reproduction et la longévité 
des vaches comme des performances à part entière, au même titre que les performances de production. Considérer l'ensemble de ces performances comme les résultantes du système d'élevage étudié constitue sans doute la meilleure approche pour évaluer globalement l'intérêt des divers traitements expérimentaux et élaborer des recommandations pertinentes pour les éleveurs.

Des relations complexes entre les facteurs expérimentaux et les divers types de performances ont ainsi été mises en évidence. Les classes de lactation les plus favorables à une bonne longévité des vaches ne sont pas celles où l'on trouve les animaux les plus productifs. Les potentiels de production les plus élevés n'ont pas conduit, dans les conditions de l'essai, aux productions les plus élevées, parce que les animaux les plus productifs se sont avérés plus exposés que d'autres aux troubles de la reproduction et aux affections cliniques diverses. La notion d'équilibre entre les diverses fonctions vitales des animaux reprend par là une certaine actualité.

\section{Références bibliographiques}

BROCHART M., FAYET J.C., 1981. Milieu et pathologie podale des vaches laitières. In : Milieu, pathologie et prévention chez les ruminants. Ed. INRA Publications, Route de Saint-Cyr, 78000 Versailles

BROSTER W.H., BROSTER V.J., 1984. Review of the progress of dairy science : long term effects of plane of nutrition on the performance of the dairy cows. J. Dairy Res. $51: 149-156$.

COULON J.B., GAREL J.P., HODEN A., JOURNET M. LIENARD G., 1985. Production laitière en zone de montagne : effets pluriannuels du type de ration hivernale et du niveau de complémentation. Bull. Tech. CRZV Theix, INRA, 61 : 31-48.

COULON J B, LANDAIS E, GAREL J.P., 1989a. Pathologie et productivité de la vache laitière : interrelations à l'échelle de la lactation. Ann. Rech. Vét. , sous presse.

COULON J.B., LANDAIS E., GAREL J.P., 1989b. Pathologie et productivité de la vache laitière: interrelations à l'échelle de la carrière. Ann. Rech. Vét., sous presse.

FAVERDIN P., HODEN A., COULON J.B., 1987, Recommandations alimentaires pour les vaches laitières. Bull. Tech. CRZV Theix, INRA, $70: 133-152$.
FAYE B., 1989. Environmental factors associated with lameness and limb disease in French dairy herds. Prev. Vet. Méd., sous presse.

FENELON J.P., 1981. Qu'est-ce que l'analyse des données? Ed. Lefonen, 311 p. Paris.

HODEN A. 1978. Rationnement en début de la lactation. In: La vache laitière. Ed. INRA Publications, Route de Saint-Cyr, 78000 Versailles.

LANDAIS E., COULON J.B., GAREL J.P., HODEN A. 1989. Caractérisation de la pathologie de la vache laitière à l'échelle de la lactation. Principaux facteurs de variation et typologie des profils pathologiques de lactation. Ann. Rech. Vét., sous presse.

LUCEY S. ROWLANDS G.J. 1984. The association between clinical mastitis and milk yield in dairy cows. Anim. Prod., 39 : 165-175

MARQUIS B., HODEN A, 1977. Répercussions de quelques ennuis sanitaires sur la production des vaches laitières. Bull. Tech., CRZV Theix, INRA, $29: 29-33$. 